\title{
The $n$-PI-Method for Helical Cone-Beam CT
}

\author{
R. Proksa*, Th. Köhler, M. Grass, and J. Timmer
}

\begin{abstract}
A new class of acquisition schemes for helical cone-beam computed tomography (CB-CT) scanning is introduced, and their effect on the reconstruction methods is analyzed. These acquisition schemes are based on a new detector shape that is bounded by the helix. It will be shown that the data acquired with these schemes are compatible with exact reconstruction methods, and the adaptation of exact reconstruction algorithms to the new acquisition geometry is described. At the same time, the so-called PI-sufficiency condition is fulfilled. Moreover, a good fit to the acquisition requirements of the various medical applications of cone-beam CT is achieved. In contrast to other helical cone-beam acquisition and reconstruction methods, the $n$-PI-method introduced in this publication allows for variable pitches of the acquisition helix. This additional feature will introduce a higher flexibility into the acquisition protocols of future medical cone-beam scanners. An approximative $n$-PI-filtered backprojection ( $n$-PI-FBP) reconstruction method is presented and verified. It yields convincing image quality.
\end{abstract}

Index Terms-Exact reconstruction, helical cone-beam computed tomography, PI-method, Radon-inversion.

\section{INTRODUCTION}

$\mathbf{R}$ ECENT developments in X-ray detector technology for medical computed tomography (CT) scanners have led to multiarray detectors and cone-beam rather than fan-beam scanning. First commercial systems are already available. The new acquisition geometry requires the adaptation of well-known two-dimensional (2-D) reconstruction methods or completely new three-dimensional (3-D) reconstruction methods. As the axial detector dimension is still limited, helical acquisition with several turns is still required to completely cover the area of diagnostic interest. First approaches toward multiarray CT reconstruction introduced preprocessing steps to interpolate data from the acquired projections and use the interpolated data in conventional or slightly modified 2-D reconstruction methods [1]-[5]. Although the approaches differ in detail, they all use approximations of the measured data to be used within the well-known 2-D-filtered backprojection method. These approximations introduce image artifacts that increase with a higher number of detector rows. For a higher number of rows, it is likely that even improved multislice approaches will fail to produce acceptable image quality for medical usage and that they have to be replaced with proper 3-D reconstruction techniques.

A serious problem with the introduction of proper 3-D reconstruction techniques for medical applications is the enor-

Manuscript received January 5, 2000; revised July 14, 2000. The Associate Editor responsible for coordinating the review of this paper and recommending it for publication was C. Crawford. Asterisk indicates corresponding author.

*R. Proksa, Th. Köhler, and M. Grass are with the Philips Research Laboratory, Division Technical Systems Hamburg, D-22335 Hamburg, Germany.

J. Timmer is with Philips Medical Systems, 5680 DA Best, The Netherlands. Publisher Item Identifier S 0278-0062(00)08356-7. mous processing effort required for these algorithms. With the increase of the number of images of multiarray CT scanners, clinical procedures will require reconstruction times of considerably less than $1 \mathrm{~s}$ per image. Standard computer systems can hardly reach this performance level today, and the dedicated hardware accelerator systems, developed for conventional 2-D helical CT, are often not applicable to the required 3-D operations.

Reviewing the cone-beam reconstruction methods known from the literature, while keeping the requirements of medical applications in mind, at least two classes of methods and related data acquisition geometries are known that might be attractive candidates for medical cone-beam CT (CB-CT), as follows.

- Exact reconstruction methods based on segmented Radon planes. These methods aim to find an analytical expression for the solution of the reconstruction problem. Various algorithms have been described. Tam et al. [6], [7] have proposed an acquisition geometry for helical CB-CT that uses a minimal detector, compatible with exact reconstruction. We call this detector shape the Tam-Danielsson window [7]-[10]. ${ }^{1}$

- Approximative 3-D-filtered backprojection methods. Various algorithms have been proposed (see, e.g., [11]-[15], and the references therein). An attractive filtered backprojection (FBP) method is the PI-method, which was invented by Danielsson and Turbell et al. [8]-[10]. It is based on the so-called PI-sufficiency condition, which requires that each object point must be illuminated by the source over an angular span of $\pi$, as seen from the point. It was shown that one solution combining a helical acquisition scheme with the fulfillment of this sufficiency condition is the usage of the Tam-Danielsson detector. Danielsson et al. have described an approximative FBP algorithm for this detector. This algorithm is simple, robust, and yields an image quality for limited cone-angles, which is acceptable for medical applications.

In this contribution, a new class of acquisition schemes for helical CB-CT scanning is introduced. The effect on the above-mentioned reconstruction methods is analyzed in detail. We describe an exact reconstruction algorithm (n-PI-EXACT) and an approximative $n$-PI-filtered backprojection reconstruction method ( $n$-PI-FBP).

This paper is organized as follows. After some definitions in Section II, a generalization of the Tam-Danielsson acquisition window, the $n$-PI-window, is introduced in Section III. In Section $\mathrm{V}$, we will show how exact or quasiexact reconstruction techniques, which are discussed in Section IV, can be adapted to the $n$-PI-window. A detailed discussion on the Radon plane segmentation and the related redundancies is given in the

\footnotetext{
${ }^{1}$ We use the term window to refer to the shape of a beam.
} 


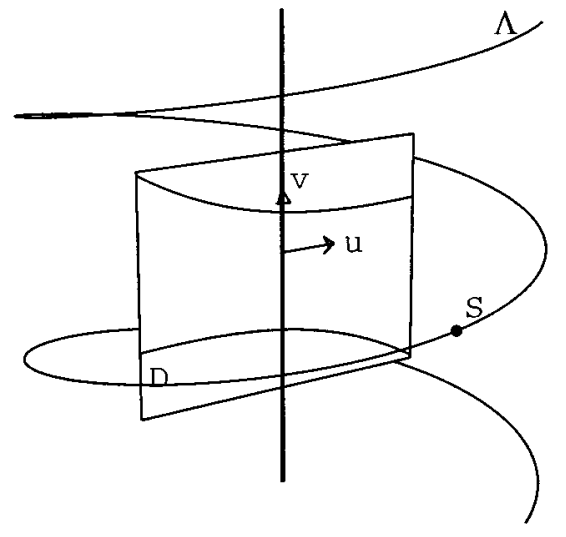

Fig. 1. Geometry used for the description of exact reconstruction algorithms. The source $S$ is moving on a helix $\Lambda$, and the virtual planar detector $D$ contains the rotation axis.

Appendix. In Sections VI and VII, the PI-method and the adaptation to the $n$-PI-geometry is discussed. The phenomenon of interrupted illumination is described in Section VIII. We prove that Danielsson's sufficiency condition is satisfied even for interrupted illumination. In Section IX, we will outline some technical problems that occur when the Tam-Danielsson window is applied to medical CT scanners. In Section X, we present simulation results and draw some conclusions for medical CB-CT scanners in Section XII.

\section{DEFINITIONS}

The reconstruction problem is to obtain the 3-D object function $f(\vec{x})$ within an object cylinder with radius $r_{\text {FOV }}$. The acquisition is modeled as measurements of line integrals of the linear X-ray attenuation coefficient $f(\vec{x})$ along lines between a point source and a virtual 2-D detector $D(u, v)$, which contains the rotation axis along $\overrightarrow{1_{v}}$ as shown in Fig. 1. The detector origin is located on this axis. The detector normal points to the source starting at $u=0, v=0$. The source-detector subsystem moves along a trajectory $\Lambda$. The source vertices are $\vec{a}(\lambda)$ for $\lambda \in \Lambda$. The resulting cone-beam projections are sets of integrals

$$
g(u, v, \lambda)=\int_{0}^{\infty} d t f(\vec{a}(\lambda)+t \vec{\beta}(u, v, \lambda)) .
$$

The unit vector $\vec{\beta}(u, v, \lambda)$ points from the source $\vec{a}(\lambda)$ toward the detector $D$ at the position $(u, v)$. The vertex of the source follows a helix with radius $R$ ( $\left.R \geq r_{\mathrm{FOV}}\right)$

$$
\vec{a}(\lambda)=(R \cos \lambda, R \sin \lambda, h \lambda) \quad \text { for } \lambda \in \Lambda .
$$

With $h$ being the slope of the helix, the pitch $P$ of the helix is $2 \pi h .^{2}$

The Radon transform [16] of $f(\vec{x})$ is defined by

$$
\mathcal{R} f(l, \vec{\xi})=\int_{R^{3}} \overrightarrow{d x} f(\vec{x}) \delta(\vec{x} \cdot \vec{\xi}-l) .
$$

${ }^{2}$ Note that we define the pitch as the table movement in the $z$-direction per rotation.
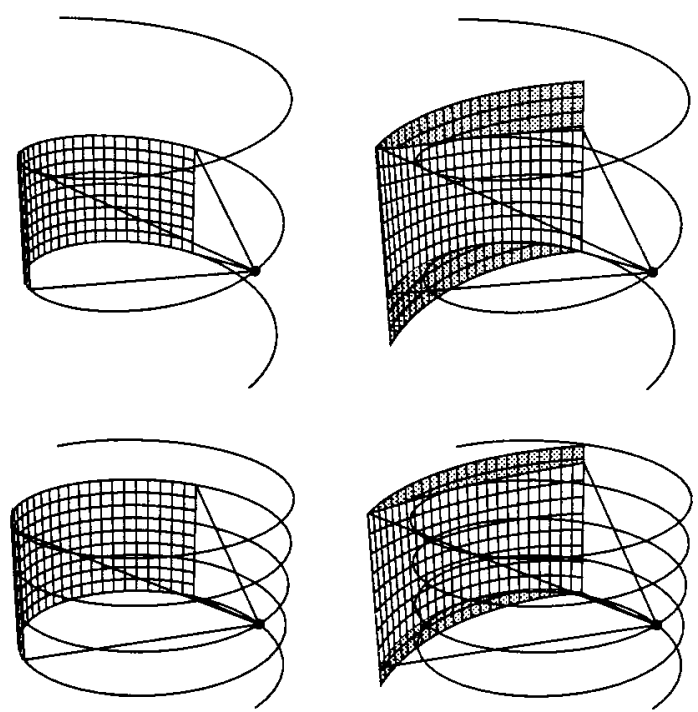

Fig. 2. Different detectors that use the PI-window (upper row) and the 3-PI-window (lower row). Left column: Detectors that are defined on the source cylinder. Right column: Detectors that are defined as part of a rectangular focus-centered detector.

The integration or Radon plane $\Pi(l, \vec{\xi})$ is normal to the unit vector $\vec{\xi} \in S^{2} / 2$ at signed distance $l$ from the origin. $S^{2} / 2$ is half the unit sphere.

\section{Generalized ACQuisition GeOMETRY}

The Tam-Danielsson window is delimited by two successive turns of the helix. The basic idea of the $n$-PI-window is to extend the detector boundaries symmetrically by one or more times the helical pitch. The detector thus covers the area defined by an odd number of source rotations. In the lower row of Fig. 2, a detector shape limited by three turns of the source path is shown. Due to the relation to the Danielsson approach, we call this window $n$-PI-window. Following this notation, the Tam-Danielsson window is the 1-PI-window.

The Tam-Danielsson window is limited by a lower and upper detector boundary defined as

$$
\begin{aligned}
\vec{U}(\lambda, s) & =\vec{a}(\lambda+\pi+s) & & \text { for }-\pi<s<\pi \\
\vec{L}(\lambda, s) & =\vec{a}(\lambda-\pi+s) & & \text { for }-\pi<s<\pi .
\end{aligned}
$$

The generalization to the $n$-PI-window has boundaries

$$
\begin{array}{ll}
\overrightarrow{U_{n}}(\lambda, s)=\vec{a}(\lambda+n \pi+s) & \text { for }-\pi<s<\pi \\
\overrightarrow{L_{n}}(\lambda, s)=\vec{a}(\lambda-n \pi+s) & \text { for }-\pi<s<\pi .
\end{array}
$$

The planar detector $D(u, v)$ is illuminated in a region $B_{n}$ defined as

$$
B_{n}=\left\{(u, v) \mid-V_{n}(-u) \leq v \leq V_{n}(u)\right\}
$$

where $V_{n}(u)$ defines the limits and is

$$
V_{n}(u)=h\left[1+\frac{u^{2}}{R^{2}}\right]\left[\frac{n \pi}{2}-\arctan \frac{u}{R}\right] .
$$

Throughout this paper, we call a data acquisition using the $n$-PI-window the " $n$-PI-method." 


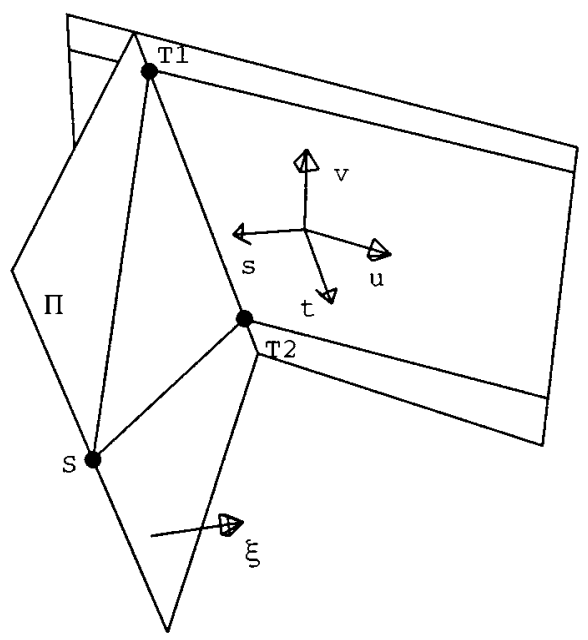

Fig. 3. Geometry used for the description of exact reconstruction algorithms. A Radon plane $\Pi$ is defined by its normal $\vec{\xi}$ and its distance $l$ (not shown) to the origin. $\Pi$ contains the source position $S$. The segment that this projection contributes to $\Pi$ is defined by the triangle $S, T_{1}, T_{2} .(u, v)$ and $(s, t)$ are the Cartesian coordinates in the detector plane corresponding to the orthonormal vectors $\left(\overrightarrow{1_{u}}, \overrightarrow{1_{v}}\right)$ and $\left(\overrightarrow{1_{s}}, \overrightarrow{1_{t}}\right)$, respectively. $\overrightarrow{1_{t}}$ is parallel to the intersection of the detector with the Radon plane $\Pi$.

\section{REVIEW ON EXACT RECONSTRUCTION METHODS}

We will briefly recall the fundamentals of exact reconstruction methods. Details on the various methods can be found, e.g., in [7], [13], and [17]-[21]. All exact reconstruction methods known to the authors are based on the Radon inversion [22]

$$
f(\vec{x})=\frac{-1}{8 \pi^{2}} \int_{0}^{\pi} d \theta \int_{0}^{2 \pi} d \varphi \frac{\partial^{2}}{\partial l^{2}} \mathcal{R} f(l, \vec{\xi}) \sin \theta
$$

with

$$
\vec{\xi}=(\sin \theta \cos \phi, \sin \theta \sin \phi, \cos \theta) .
$$

A fundamental problem is that the required Radon values cannot be extracted from the measured data directly. This is because the rays in a plane are divergent and a simple integration of the line integrals would not yield the required plane integral. An important contribution to this problem was Grangeat's fundamental relation [17], [21], [23], which shows how the first derivative of the Radon transform with respect to the distance $l$ of the plane to the origin can be calculated from the divergent rays

$$
\begin{aligned}
\mathcal{R}^{\prime} f(l, \vec{\xi}) & =\frac{\partial}{\partial l} \mathcal{R} f(l, \vec{\xi}) \\
& =\frac{1}{\cos ^{2} \beta} \int_{-\infty}^{\infty} d t \frac{\partial}{\partial s} \tilde{g}(s, t, \lambda)
\end{aligned}
$$

with $\beta$ being the angle between the Radon plane $\Pi$ and the detector normal $\overrightarrow{1_{u}} \times \overrightarrow{1_{v}} \cdot \tilde{g}(s, t, \lambda)$ is a rotated version of $(R / d) g(u, v, \lambda)$ such that $\overrightarrow{1_{t}}$ is parallel to the intersection of the detector plane with $\Pi$. $\overrightarrow{1_{s}}$ is normal to $\overrightarrow{1_{t}}$ and on the detector, and $d$ is the distance of the source $S$ to a particular point $(u, v)$ on the detector. Please note that $\tilde{g}(s, t, \lambda)$ depends on $l$ and $\vec{\xi}$, as shown in Fig. 3 .

This intermediate result is the key to exact reconstruction methods. The remaining problem is the reconstruction from the derivative of the 3-D Radon transform. Several algorithms have
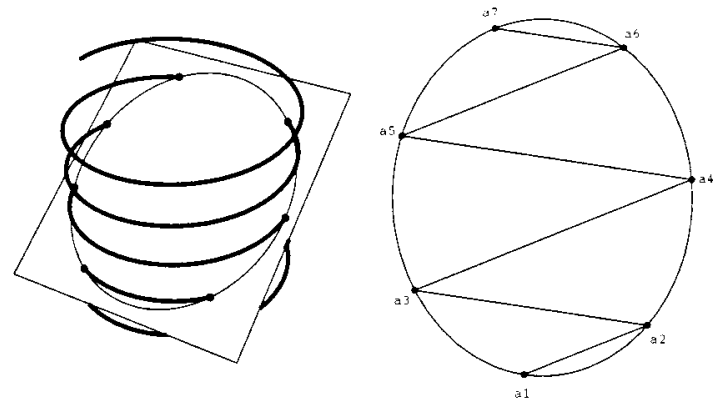

Fig. 4. Example for a triangulation of a Radon plane. Left: A Radon plane intersecting the helix, resulting in seven intersection points. The part of the Radon plane lying inside the source cylinder is an ellipse. Right: View perpendicular to the Radon plane onto the ellipse. The lines connecting the source positions $a_{i}$ indicate the lines $a_{i} T_{1}$ and $a_{i} T_{2}$. The Radon plane is filled completely and nonredundantly by the resulting segments.

been proposed to solve this problem. They differ mainly in their numerical stability and computational complexity. An overview of the various techniques can be found in [11] and [12] and the references therein. Almost all techniques generate the derivative of the Radon transform as an intermediate result. As we will see later, this is important for the adaptation of these methods to the $n$-PI-geometry. The cone-beam FBP algorithm from Defrise and Clack [18] and Kudo and Saito [24] is one exception. This algorithm, called the Defrise-Kudo method here, was derived from the same mathematical framework, but uses a different formulation of the various operations involved. An important difference is that the derivative of the Radon transform does not need to be calculated explicitly on a $(l, \vec{\xi})$-grid. Defrise has introduced a variable exchange $(l \rightarrow \lambda)$ with $\vec{a}(\lambda) \cdot \vec{\xi}=l$ and can perform the final backprojection step over the source trajectory $\lambda$. Because $\vec{a}(\lambda) \cdot \vec{\xi}=l$ can have multiple solutions, a normalization is required. In its simplest form, this normalization function is just the inverse of the number of solutions of $\vec{a}(\lambda) \cdot \vec{\xi}=l$, which is equivalent to the number of redundant measures for a Radon value. In our notation, the normalization factor becomes

$$
M(l, \lambda)=\frac{1}{N_{D}(l, \lambda)}
$$

with $N_{D}$ being the number of nontruncated illuminations of a Radon plane. If the acquisition fulfills the Tuy-Smith condition [25], [26], $N_{D}$ is greater than zero for all Radon planes intersecting the object. Because this $M$-function might be discontinuous, a smoothed differentiable version of $M$ was used by Defrise.

All algorithms mentioned so far were developed for axially nontruncated projections. A first solution for truncated projections from a helical trajectory was described by Tam et al. [7] based on a special window (Tam-Danielsson window, as mentioned in Section I) that provides complete and quasi nonredundant data capture in the Radon domain. The lower and upper boundary of the detector is defined by the vertex path. The intersection of this beam geometry with an arbitrary Radon plane defines triangle-like segments. The individual segments of all source intersections fill the Radon plane completely and in a quasi nonredundant way. An example is shown in Fig. 4, where all triangle-like segments are illuminated from 
one source position each. As an example: when the source is at position $\vec{a}\left(\lambda_{4}\right)$, the related detector borders in this plane are at $\vec{a}\left(\lambda_{3}\right)$ and $\vec{a}\left(\lambda_{\tilde{5}}\right)$. The related segment is exclusively illuminated from source vertex $\vec{a}\left(\lambda_{4}\right)$. All segments together completely cover the ellipse.

The main idea of the exact reconstruction approach for truncated projections is to combine the partial contributions of each segment $\Delta_{i}$ to the derivative of the Radon transform. The integration is reduced to the measured interval ${ }^{3}\left[t_{1}, t_{2}\right]$

$$
\mathcal{R}_{\Delta_{i}}^{\prime} f(l, \vec{\xi}) \approx \frac{1}{\cos ^{2} \beta} \int_{t_{1}}^{t_{2}} d t \frac{\partial}{\partial s} \tilde{g}(s, t, \lambda) .
$$

The summation of all $N_{\Delta}$-contributions leads to the required Radon derivative

$$
\mathcal{R}^{\prime} f(l, \vec{\xi})=\sum_{i=1}^{N_{\Delta}} \mathcal{R}_{\Delta_{i}}^{\prime} f(l, \vec{\xi})
$$

Based on this framework, different reconstruction methods are known to solve the remaining problem of calculating an object function from the first derivative of the Radon transform.

Kudo et al. [27] have shown that the correct summation of the partial components requires an additional term for the borders of the integration intervals

$$
\begin{aligned}
\mathcal{R}_{\Delta_{i}}^{\prime} f(l, \vec{\xi})= & \frac{1}{\cos ^{2} \beta} \int_{t_{1}}^{t_{2}} d t \frac{\partial}{\partial s} \tilde{g}(s, t, \lambda)-\frac{\tan \beta}{R} \\
& \cdot\left(t_{1} \tilde{g}\left(s, t_{1}, \lambda\right)-t_{2} \tilde{g}\left(s, t_{2}, \lambda\right)\right) .
\end{aligned}
$$

The Defrise-Kudo algorithm was reformulated by Kudo et al. [27] for the segmented helical approach.

\section{Adaptation of the Exact Algorithm for the $n$-PI-CASE}

We will now adapt the exact reconstruction methods discussed in the previous section to the $n$-PI-acquisition geometry. As for the PI-case, the basis of an exact reconstruction method is a proper triangulation of the Radon planes. The intersection of a plane $\Pi(l, \vec{\xi})$ with the object cylinder is an ellipse $E$. On this ellipse, we have an odd number $n_{0}$ of intersections with the vertex path. For the $n$-PI-case, we have the following segmentation:

- For all planes $\Pi(l, \vec{\xi})$ with $n_{0}$-intersections and $n_{0}<n$, the plane is completely covered $n_{0}$-times. We have a constant and homogeneous overrepresentation of this Radon plane.

- For all planes $\Pi(l, \vec{\xi})$ with $n_{0}$-intersections and $n_{0} \geq n$, the plane is completely covered $n$-times. We have again a constant overrepresentation.

A detailed proof and discussion of this segmentation is given in the Appendix. As already observed by Kudo et al. [27] for the PI-case, a slight redundancy of the plane coverage is also present in the $n$-PI-case. Due to this, we call the acquisition quasinonredundant. The constant overrepresentation of the Radon planes can be treated with a normalization. In the Appendix, this

${ }^{3}$ For the sake of compactness, we do not explicitly write the dependency of $t_{1}$ and $t_{2}$ on $s$. normalization is given in terms of a proper $M$-function, which is the inverse of the number of overrepresentations. The result is

$$
M_{n}(l, \vec{\xi}, \lambda)= \begin{cases}\frac{1}{n_{0}(l, \vec{\xi})}, & n_{0}(l, \vec{\xi})<n \\ \frac{1}{n}, & \text { otherwise. }\end{cases}
$$

For all exact reconstruction methods that explicitly calculate the derivate of the Radon transform, the adaptation to the $n$-PI-case is the normalization of this intermediate result with the $M$-function. The complete $n$-PI-EXACT algorithm is

- For all $\Pi(l, \vec{\xi})$, calculate $\mathcal{R}^{\prime} f(l, \vec{\xi})$ by

- Determine $n_{0}$

- For all $n_{0}$-intersections

- Determine $\vec{a}\left(\lambda_{i}\right)$

- Determine the integration interval $t_{1}$ and $t_{2}$

- Calculate

$$
\begin{aligned}
\mathcal{R}_{\Delta_{i}}^{\prime} f(l, \vec{\xi})= & \frac{1}{\cos ^{2} \beta} \int_{t_{1}}^{t_{2}} d t \frac{\partial}{\partial s} \tilde{g}\left(s, t, \lambda_{i}\right)-\frac{\tan \beta}{R} \\
& \cdot\left(t_{1} \tilde{g}\left(s, t_{1}, \lambda_{i}\right)-t_{2} \tilde{g}\left(s, t_{2}, \lambda_{i}\right)\right)
\end{aligned}
$$

- Calculate

$$
M=\frac{1}{\min \left(n, n_{0}\right)}
$$

- Calculate

$$
\mathcal{R}^{\prime} f(l, \vec{\xi})=M \sum_{i=1}^{n_{0}} \mathcal{R}_{\Delta_{i}}^{\prime} f(l, \vec{\xi})
$$

- Calculate

$$
f(\vec{x})=\frac{-1}{4 \pi^{2}} \int_{S^{2} / 2} \frac{\partial}{\partial l} \mathcal{R}^{\prime} f(l, \vec{\xi}) .
$$

The adaptation of the Defrise-Kudo-style exact algorithms must be treated in a different way. Most of the processing is done directly on the detector data before the final backprojection step over the source trajectory $\Lambda$ is done. Because the Defrise-Kudo algorithm explicitly uses an $M$-function normalization, we can use the $n$-PI-definition of the $M$-function (17). As described by Defrise, the simple version of the $M$-function defined as $M=$ $1 / N_{D}$ is discontinuous and yields problems in a derivation step after its application. Defrise derived a smoothed version $\tilde{M}$ of $M$ such that the required normalization is satisfied

$$
\sum_{i=1}^{N_{D}} \tilde{M}\left(l, \vec{\xi}, \lambda_{i}\right)=1,
$$

but the individual values $M\left(l, \vec{\xi}, \lambda_{i}\right)$ may be different. For the $n$-PI-case, we have to use an additional constraint on $\tilde{M}$ to guarantee the homogeneous weighting over the Radon plane. Because we construct the planes from overlapping segments, we cannot allow all segments to be weighted differently. However, we can split the segmentation of a plane into a superposition of subsegments. Each of them segments the entire plane completely and quasi-nonredundantly. A simple algorithm to perform this subsegmentation is the construction of the subsegments according to

$$
\Omega_{l}=\left\{\vec{a}\left(\lambda_{j}\right) \mid j=l+k n, k \in N, l+k n \leq n_{0}\right\}
$$




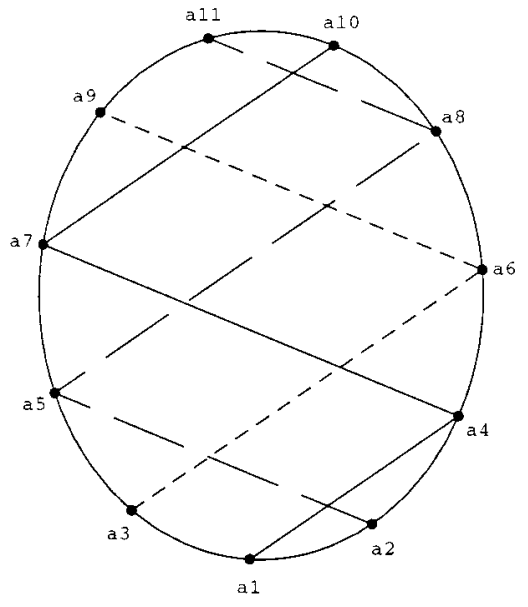

Fig. 5. Example of a subsegmentation of a Radon plane in the 3-PI-mode. The three subsegments are $\left\{\vec{a}\left(\lambda_{1}\right), \vec{a}\left(\lambda_{4}\right), \vec{a}\left(\lambda_{7}\right), \vec{a}\left(\lambda_{10}\right)\right\},\left\{\vec{a}\left(\lambda_{2}\right), \vec{a}\left(\lambda_{5}\right)\right.$, $\left.\vec{a}\left(\lambda_{8}\right), \vec{a}\left(\lambda_{11}\right)\right\}$, and $\left\{\vec{a}\left(\lambda_{3}\right), \vec{a}\left(\lambda_{6}\right), \vec{a}\left(\lambda_{9}\right)\right\}$.

for $l=1, \cdots, n . N$ are the natural numbers. $\Omega_{l}$ are sets of vertex positions that must be weighted identically. An example of a subsegmentation is shown in Fig. 5.

Each subsegment is constructed from vertex positions with an index difference of $n$ between successive vertices. This results in a segmentation as derived from a PI-scan with a helical pitch $n$-times larger than the $n$-PI-scan under consideration. This provides a complete and quasi-nonredundant segmentation. If each component of a subsegment gets the same $\tilde{M}$-function weighting, different weightings of the superposition of the individual subsegments can be allowed to get $\tilde{M}$. Our additional constraint is ${ }^{4}$

$\tilde{M}\left(l, \vec{\xi}, \lambda_{i}\right)=\tilde{M}\left(l, \vec{\xi}, \lambda_{i+j n}\right) \quad$ for $j \in N, i+j n \leq n_{0}$.

The adapted Defrise-Kudo constraint for the $n$-PI-case is

$$
\sum_{i=1}^{\min \left(n, n_{0}\right)} \tilde{M}\left(l, \vec{\xi}, \lambda_{i}\right)=1 .
$$

For the PI-case, $\tilde{M}$ is usually set to 1 because all Radon planes are completely and quasi nonredundantly covered once.

Adapted versions of the Defrise-Kudo algorithm, such as described in Kudo et al. [27], have been simplified by using a constant $M$-function. It remains to reformulate this algorithm with the $n$-PI $\tilde{M}$-function.

\section{REVIEW ON THE PI-METHOD}

In the next section, we will describe an FBP-style algorithm for the $n$-PI-method that can be seen as a generalization of the PI-method. We will briefly review this method. The PI-method is based on the sufficiency condition that requires that an object point must be illuminated over an angular range of exactly $\pi$, as seen from the object point [8], [28]. Fig. 6 shows a projection ${ }^{4}$ In the example shown in Fig. 5 , every $\tilde{M}$ could be set to $M_{1}$ for
$\lambda_{1}, \lambda_{4}, \lambda_{7}, \lambda_{10}$, to $M_{2}$ for $\lambda_{2}, \lambda_{5}, \lambda_{8}, \lambda_{11}$, and $1-M_{1}-M_{2}$ for $\lambda_{3}, \lambda_{6}, \lambda_{9}$

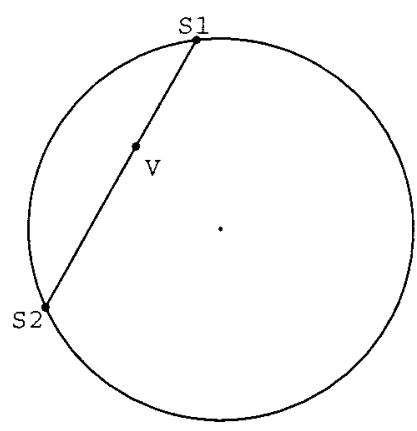

Fig. 6. The helix as seen along the rotation axis, a vertex position $S_{1}$, and an object point $V$ that just entered the cone. According to the PI-sufficiency condition, $V$ must leave the cone if the vertex is at position $S_{2}$.

of the acquisition geometry along the rotation axis. An arbitrary object point $V$ that just enters the cone when the source is at position $S_{1}$ (and is projected onto the detector element at $S_{2}$ ) must leave the cone if the source has changed its position to $S_{2}$ and the related detector border is at position $S_{1}$. The Tam-Danielsson window used in the PI-method fulfills this requirement. For this detector, the vertex has moved from $S_{1}$ to $S_{2}$ over a range of $\Delta \lambda$, where

$$
0 \leq \Delta \lambda \leq 2 \pi,
$$

depending on the position of the object point. Danielsson et al. call such a line $\left(S_{1} \rightarrow S_{2}\right)$ a PI-line.

The first step of the reconstruction is a parallel rebinning [10], [29], row by row to a virtual planar detector containing the rotation axis. Due to the detector geometry, the area intersected by the rebinned projections on this virtual detector is a perfect rectangle [8]-[10]. The rebinned data are weighted, filtered row-wise and backprojected.

Note that (25) becomes

$$
2 \pi / 3 \leq \Delta \lambda \leq 4 \pi / 3
$$

if we assume a realistic maximum fan-angle of $\pm 30^{\circ}$. Therefore, the illumination time of object points varies over the field of view (FOV) by a factor of two.

An important aspect of the PI-method is the concept of PI-lines. As mentioned above, a PI-line is a connection of the source and the detector border. All object points along this line will enter and leave the cone-beam simultaneously. We will use the PI-line concept later to discuss the proposed $n$-PI-window.

\section{THE $n$-PI-FBP-METHOD}

If we consider the detector described in Section III, the PI-sufficiency condition is still fulfilled, but using the same notation as in the previous section, the vertex has moved at least $(n-1) / 2$ full turns

$$
(n-1) \pi \leq \Delta \lambda \leq(n+1) \pi .
$$

If the fan angle is again restricted to $\pm 30^{\circ}$, this becomes

$$
(n-1) \pi+2 \pi / 3 \leq \Delta \lambda \leq(n+1) \pi-2 \pi / 3 .
$$




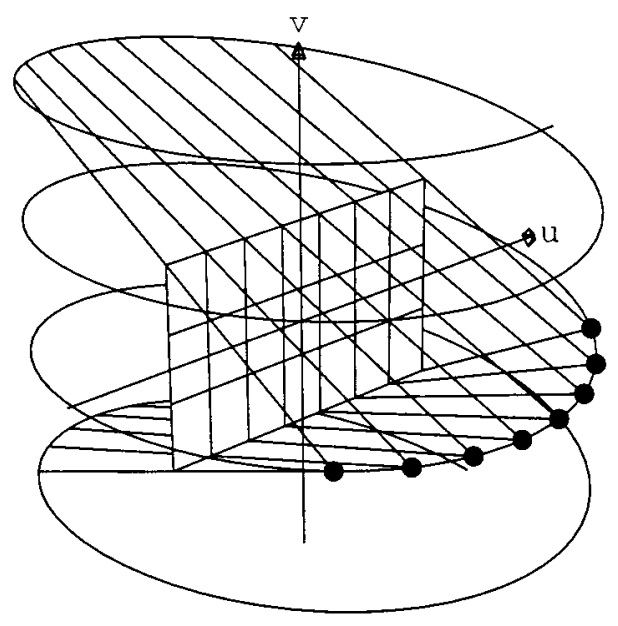

Fig. 7. The beam geometry after parallel rebinning for the 3-PI-case. The rays intersect the $(u, z)$-plane in a rectangle.

The resulting variation in the illumination time is then

$$
\frac{\Delta \lambda_{\max }}{\Delta \lambda_{\min }}=\frac{3 n+1}{3 n-1}
$$

For the 3-PI-mode, this is 1.25 .

We propose to use a similar FBP algorithm for the $n$-PIwindow as for the PI-window with a slight adaptation to the different pitch during the backprojection. Therefore, the reconstruction is performed as follows ${ }^{5}$ :

1) Parallel rebinning of the cone-beam data. Each row is treated the same way. The new projection angle $\theta$ and the new column coordinate $u$ are related to the vertex position $\lambda$ and the fan-angle $\gamma$ according to

$$
\theta=\lambda+\gamma, \quad u=\sin \gamma
$$

This leads to the geometry shown in Fig. 7. The virtual detector is a rectangle as shown in the Appendix.

2) Depending on the pixel spacing on the real detector, a resampling on the virtual detector is necessary to get a rectangular sampling grid on the virtual detector.

3) Preweighting of the rebinned data with the cosine of the cone-angle

$$
\cos \kappa=\frac{\sqrt{1-u^{2}}}{\sqrt{1-u^{2}+(\gamma+v)^{2}}}
$$

and ramp filtering row by row.

4) Backprojection of the filtered data using the rebinned cone-beam geometry. The backprojection can be described in a system with a fixed extended virtual source at

$$
\vec{s}=\left(x, \sqrt{1-x^{2}}, \arcsin x\right) .
$$

${ }^{5} \mathrm{We}$ assume that the detector lies on the source cylinder as shown in the left column of Fig. 2, and we use a scaled coordinate system with $R=1$ and $P=2 \pi$ to get compact formulas.
The virtual detector lies in the $x z$-plane with detector coordinates $u$ and $v$. A voxel at position $\left(p_{x}, p_{y}, p_{z}\right)$ is projected onto the detector at

$$
\begin{aligned}
& u=p_{x}, \\
& v=\arcsin p_{x}+\frac{p_{z}-\arcsin p_{x}}{1-\frac{p_{y}}{\sqrt{1-p_{x}^{2}}}} .
\end{aligned}
$$

Due to the acquisition geometry, it is ensured that every object point receives contributions from the virtual source over an angular range of $n \pi$. Therefore, no additional normalization is necessary. Because the acquisition geometry fulfills the PI-sufficiency condition, the name $n$-PI-method is thereby justified.

At this point, we like to stress the point that neither the PI-FBP method nor the $n$-PI-FBP method are exact. Even if the acquisition fulfills the $\pi$ sufficiency condition, the algorithm is an approximation.

We extend the notation of PI-lines to $n$-PI-lines for the $n$-PIcase. Due to the sufficiency condition in which we analyze the acquisition as seen from the object point, we will call the events when an object point enters or leaves the cone-beam sunrise and sunset.

If we analyze the data acquisition, we observe that each point has had a sunrise and a sunset event on an $n$-PI-line. Within the illumination path, each point has $(n-1 / 2)$ complete additional $\pi$-illumination intervals bounded by PI-lines, namely, PI-, 3-PI-, 5 -PI-, $\cdots$, and the $(n-2)$-PI-line. The reason is that a $(n-$ 2 )-PI-window is completely contained within a $n$-PI-window. This is a consequence of the fact that a $(n-2)$-PI-detector is completely contained within a $n$-PI-detector, as can be seen in Fig. 2.

Danielsson calls the set of all PI-lines belonging to the same projection a PI-surface. We want to extend this notation to upper and lower $n$-PI-surfaces $S_{n}^{U}(\vec{a}(\lambda))$ and $S_{n}^{L}(\vec{a}(\lambda))$.

\section{INTERRUPTED ILLUMINATION}

In this section, we will discuss a phenomenon that can occur in CB-CT with a small helical pitch compared with the detector size in $z$-direction.

Considering an object point in its relative movement to the source-detector system for helical trajectories, this object point comes into the $\mathrm{CB}$, is illuminated for some time, and leaves the $\mathrm{CB}$ forever when using the 1-PI-window. In contrast, for the $n$-PI-window with $(n \geq 3)$, an object point can enter and leave the CB several times before leaving the CB forever. An example for the 3-PI-window is shown in Fig. 8 visualizing a fixed virtual detector (after parallel rebinning) with a line source and a moving object point that follows a helical path. The same illumination path projected onto the unit sphere centered at the object point is presented in Fig. 9.

The fact that an object point enters and leaves the cone as shown in this figure is an effect connected to its complete illumination path. The consideration of static Radon planes, which forms the basis of exact reconstruction methods, is not affected. This is even true if the final backprojection is an integration over the source trajectory, as in the Defrise-Kudo-style algorithms. 

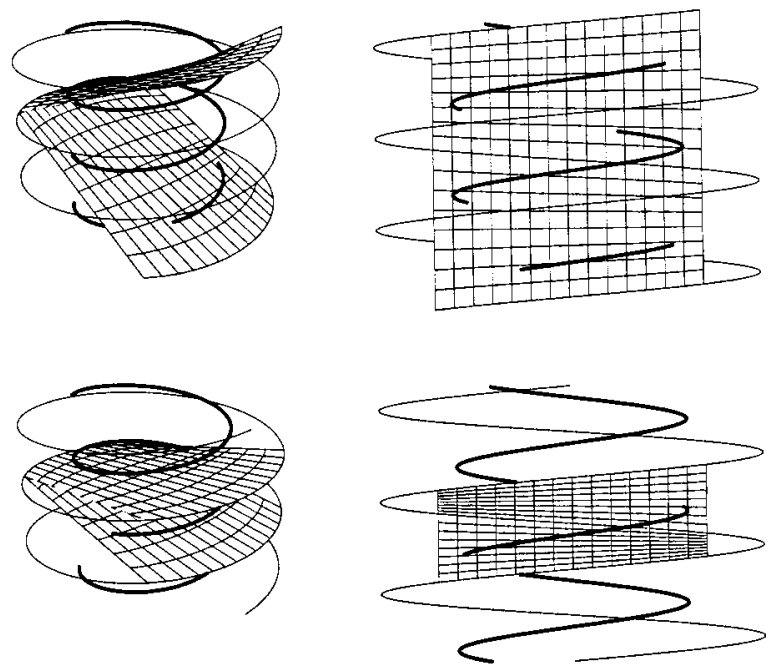

Fig. 8. Example for an object point that moves into and out of the cone several times (upper row). Left: Three-dimensional view. The object point is considered to move on a helical path, shown as a thick helix, through a stationary source-detector system. The rebinned beam geometry is displayed as the lower and upper beam boundaries. Right: View from the opposite of the source. It can be clearly seen that the lower illuminated part of the object points path is complementary to the missing part in the upper half of the picture. In the lower row, the same object point is placed into a PI-window.

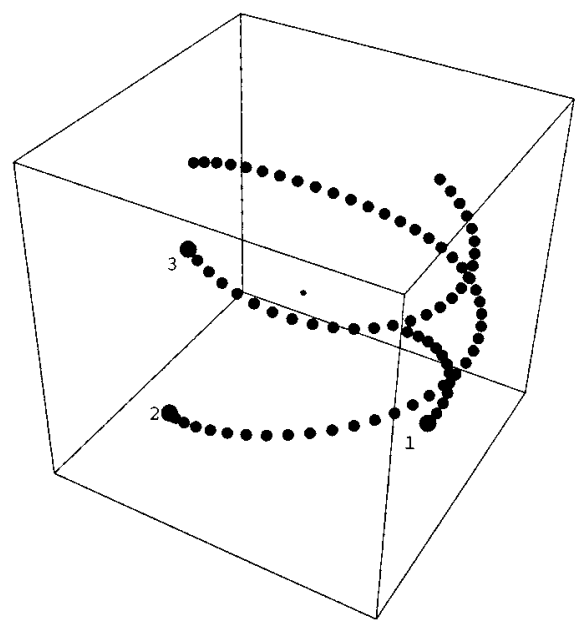

Fig. 9. Illumination path for an object point with an interrupted illumination. The small dot in the center of the cube indicates the object point, and the other dots correspond to the positions of the vertex projected onto the unit sphere centered around the object point. The positions of sunrise events are indicated by fat dots, which are also labeled according to their occurrence in time.

However, the sufficiency condition of Danielsson could be effected, because the intention of the PI-sufficiency condition is that a continuous illumination path between sunrise and sunset exists. Fortunately, continuous illumination does not necessarily mean continuous in time, a requirement that is definitely not fulfilled. The important requirement is a continuous illumination over the required angular interval, which can be measured at arbitrary times or time intervals. As we will show, the illumination intervals can always be combined to yield a complete illumination path.

For a projection in the $n$-PI-window, there is one central plane orthogonal to the rotation axis that includes the source

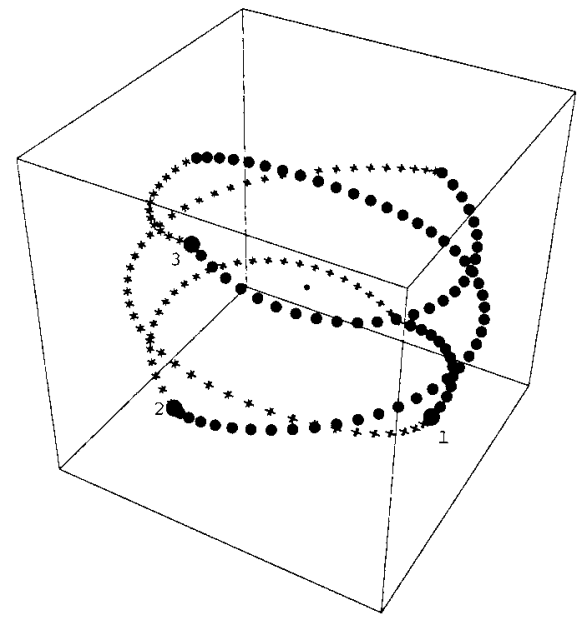

Fig. 10. As in Fig. 9, including the complementary vertex positions that are indicated by crosses. Note that sunrise 1 is complementary to sunset 2 , sunrise 2 is complementary to sunset 3 , and sunrise 3 is complementary to sunset 1 . Therefore, the second, the third, and the complementary of the first segment form a closed line that fulfills the PI-sufficiency condition.

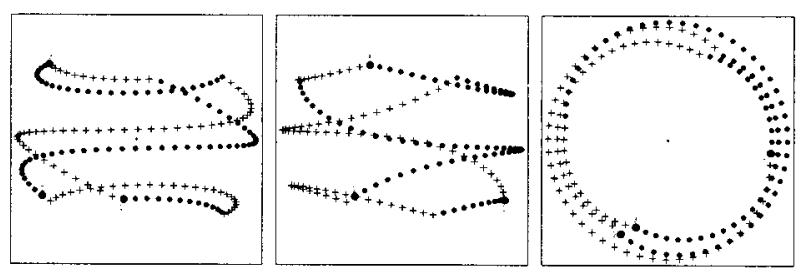

Fig. 11. As in Fig. 10, but seen along the three main axes.

that subdivides the cone in a lower and an upper part. Each point crossing that plane is in the $\mathrm{CB}$. Consequently, there is always an odd sum of sunrise and sunset events on the lower and upper $n$-PI-surfaces $\left(S_{n}^{U}\right.$ and $S_{n}^{L}$ ). Moreover, an object point is on the same $n$-PI-line when it enters and leaves the CB. Because the path of a given object point always has a constant slope relative to a CB entry point, all sunrise and related sunset points are always on opposite $n$-PI-surfaces. Putting these observations together, we can state that there will be a number of nonilluminated angular intervals below the central plane. For each of these intervals, we have a sunset and a sunrise event on a $n$-PI-surface. Due to the symmetry, we have exactly related sunrise and sunset events on the opposite $n$-PI-surface. Hence, each nonilluminated angular interval appearing below the central plane will be compensated by a corresponding illuminated interval above the central plane, and vice versa.

The compensated intervals are complementary with exchanged source and related detector positions. Because the result of the data acquisition (1) does not depend on the integration direction, we will get the same results. This change becomes visible as a sudden change of the illumination path on the unit sphere; see Figs. 10 and 11. In Section X, we show that the interrupted illumination will not degrade the resulting image quality. The phenomenon happens in $n$-PI-windows $(n \geq 3)$ for object points with a certain distance from the rotation axis. This critical radius is discussed in the Appendix. 


\section{Problems of the TAM-DANiElsSon Window FOR CT SCANNERS}

If the Tam-Danielsson window and the related acquisition scheme is applied to a feasible 2-D detector for medical CT scanners, some practical problems appear. The following list of problems is not complete.

In conventional helical CT scanning with a one-dimensional (1-D) detector, the pitch of the helix serves as a free scan parameter. It is used to optimize the balance between signal-tonoise ratio (SNR), slice thickness, X-ray dose, and scan time. On average, it is 1 for clinical applications. When using the Tam-Danielsson window, the pitch is no longer a free parameter. The fixed relation of patient-feed per rotation to detector size results in a short scan method, equivalent to a pitch of 2 in conventional CT, which is too high for some applications.

The system geometry is very effective in the sense that a large volume is scanned per rotation of the source-detector subsystem. With the current limitations of the maximum power of X-ray tubes, this leads to an insufficient SNR in the resulting images for most applications. The increase in the X-ray tube output would be costly and technically hard to achieve.

Another problem is related to the inhomogeneous illumination time, which depends on the position of object points. It varies by a factor of about 2 within the FOV for typical fan-angles; see (26). This leads to a nonuniform angular sampling density.

For technical reasons and the need for circular scanning, it is likely that a real detector would have a rectangular shape on a cylinder centered around the focal spot of the X-ray tube. Projecting the Tam-Danielsson window shape to such a detector with a fan-angle of $\pm 25^{\circ}$ reveals an ineffectively used detector area. Only about $73 \%$ of the area is used.

\section{Results}

\section{A. Practical Improvements}

The problems of the Tam-Danielsson window, as stated in Section IX, are reduced for the $n$-PI-window. Because the object points are illuminated longer, the difference between the shortest and longest illumination time is reduced; see (29). The same holds true for the variation in the angular sampling density.

The longer illumination time prevents excessive X-ray tube power requirements for medical low contrast applications that need a high SNR. The strong variation of about $100 \%$ for the PI-window is reduced to only $25 \%$ for the 3-PI-window.

The spatial efficiency of the projection of the required window onto a rectangular detector will increase from $73 \%$ to at least $86 \%$ for the 3-PI-case.

Finally, the helical pitch can be chosen in discrete steps being optimized to the medical application under consideration.

\section{B. Simulation Results}

In this subsection, we show simulation results for the new n-PI-FBP algorithm and compare them to the PI-FBP algorithm. Two different detectors were used in the simulation, one with 16 arrays, which seems to be feasible in the near future for medical
TABLE I

GEOMETRY OF THE TwO SCANNERS AND CORRESPONDING SCAN PARAMETERS USED FOR Simulation ( $S D$ : Distance SOURCE-DETECTOR, $R$ : HeliX RADIUS, $H$ : DETECTOR HEIGHT)

\begin{tabular}{c||c|c}
\hline & Det16 & Det64 \\
\hline \# rows & 16 & 64 \\
\hline \# columns & 512 & 512 \\
\hline \#ertices/turn & 1440 & 1440 \\
\hline$H$ & $1000 \mathrm{~mm}$ & $1000 \mathrm{~mm}$ \\
\hline$H$ & $500 \mathrm{~mm}$ & $500 \mathrm{~mm}$ \\
\hline fan-angle & $58.7^{\circ}$ & $58.7^{\circ}$ \\
\hline cone-angle & $1.83^{\circ}$ & $7.32^{\circ}$ \\
\hline pitch for $n=1$ & $21 \mathrm{~mm}$ & $83 \mathrm{~mm}$ \\
\hline pitch for $n=3$ & $8.3 \mathrm{~mm}$ & $33 \mathrm{~mm}$ \\
\hline pitch for $n=5$ & $5.3 \mathrm{~mm}$ & $21 \mathrm{~mm}$ \\
\hline
\end{tabular}

CT scanners, and one with 64 arrays of the same pixel size to demonstrate the high potential of the proposed method for larger cone-angles. Details of the scanner geometries and the scan parameters are summarized in Table I. A modified Shepp-Logan phantom was used. It was scaled to just fit inside the FOV of 490-mm diameter. The scaling was done to investigate whether there is an image degradation in regions with interrupted illumination. We added some high-contrast ellipsoids, because strong contrasts in the $z$-direction produce severe artifacts, which are well visible in the sagittal views. These additional objects are useful for the verification and benchmarking of $\mathrm{CB}$ reconstruction algorithms. The data of the unscaled phantom are given in Table II.

Projection data were simulated analytically on a focus-centered detector. The detector pixel size was $2 \times 2 \mathrm{~mm}^{2}$ on the real detector, corresponding to $1 \times 1 \mathrm{~mm}^{2}$ on the virtual detector in the center of rotation. For each detector, three data sets with a pitch corresponding to the 1-PI-, 3-PI-, and 5-PI-window were calculated. Reconstruction was performed using a bandlimited ramp filter [30].

The images displayed in Figs. 12-15 were reconstructed on a grid with isotropic resolution of $1 \mathrm{~mm}^{3}$.

Fig. 12 shows mid-sagittal slices, whereas Fig. 13 shows axial slices for the 16 array detector. Note the small contrast window of $10 \mathrm{HU}$, which is $0.5 \%$ of the full contrast range of the entire image and $5 \%$ of the contrast between the inner background and the high contrast spheres. Even for this small cone-angle, the PI-method shows its typical arc-shaped artifacts, which disappear with increasing PI-modes.

For the large cone-angle case, as shown in Figs. 14 and 15, image quality is significantly improved with higher PI-mode. Note the dark shading behind the high-contrast spheres in the upper left corner of the images in Fig. 14, which are clearly reduced in the 3-PI- and 5-PI-modes, and the arc-shaped artifacts in the uppermost image in Fig. 15. 
TABLE II

Definition of the Modified ShepP-Logan Phantom Used for the SimUlations. For Calculation of Hounsfield Units, We Assume $\mu_{\text {watcr }}=0.0183$

\begin{tabular}{c||c|c|c|c}
\hline no. & center & $(\theta, \phi)$ & half axes & density \\
\hline \hline 1 & $(0,0,0)$ & $(0,0)$ & $(6.9,9.2,9.0)$ & 0.0366 \\
\hline 2 & $(0,-0.184,0)$ & $(0,0)$ & $(6.624,8.74,8.8)$ & -0.017934 \\
\hline 3 & $(0.6,-1.05,-6.25)$ & $(0,0)$ & $(0.4,0.56,1.0)$ & 0.000366 \\
\hline 4 & $(0.0,1.0,-6.25)$ & $(0,0)$ & $(0.56,0.56,1.0)$ & -0.000366 \\
\hline 5 & $(-2.2,0.0,2.5)$ & $(-72,0)$ & $(4.1,1.6,2.1)$ & -0.000366 \\
\hline 6 & $(2.2,0.0,2.5)$ & $(72,0)$ & $(3.1,1.1,2.2)$ & -0.000366 \\
\hline 7 & $(0.0,3.5,2.5)$ & $(0,0)$ & $(2.1,2.5,3.5)$ & 0.000183 \\
\hline 8 & $(0.0,1.0,2.5)$ & $(0,0)$ & $(0.46,0.46,0.46)$ & 0.000183 \\
\hline 9 & $(0.0,-1.0,2.5)$ & $(0,0)$ & $(0.46,0.46,0.46)$ & 0.000183 \\
\hline 9 & $(-0.8,-6.05,2.5)$ & $(0,0)$ & $(0.46,0.23,0.2)$ & 0.000183 \\
\hline 10 & $(0.6,-6.05,2.5)$ & $(0,0)$ & $(0.23,0.46,0.2)$ & 0.000183 \\
\hline 11 & $(0.0,-6.05,2.5)$ & $(0,0)$ & $(0.23,0.23,0.23)$ & 0.000183 \\
\hline 12 & $(-0.8,-8.2,0.35)$ & $(0,0)$ & $(0.46,0.23,0.2)$ & 0.00366 \\
\hline 13 & $(0.6,-8.8,0.35)$ & $(0,0)$ & $(0.23,0.46,0.2)$ & 0.00366 \\
\hline 14 & $(0.0,-8.2,0.35)$ & $(0,0)$ & $(0.23,0.23,0.23)$ & 0.00366 \\
\hline
\end{tabular}
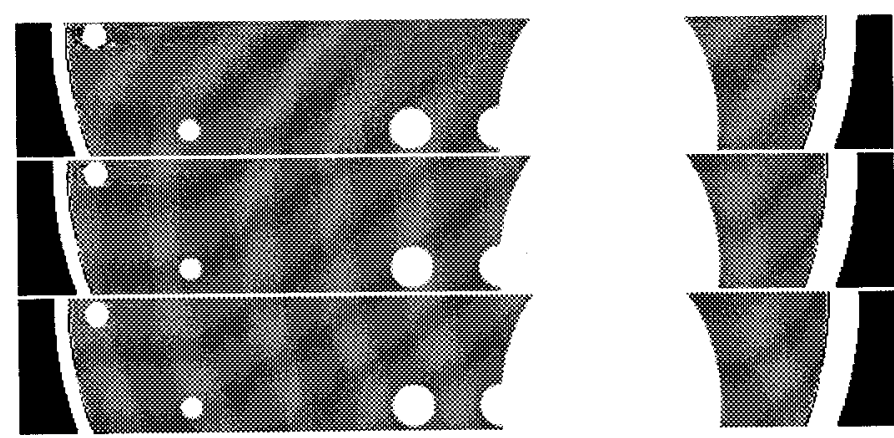

Fig. 12. Mid-sagittal slice through the reconstructed Shepp-Logan phantom for the 16 array detector using the $n$-PI-FBP-method in 1-PI-mode (top), 3-PI-mode (middle), and 5-PI-mode (bottom). Level: $20 \mathrm{HU}$; window: $10 \mathrm{HU}$.

It is interesting that both types of artifact also show up in other 3-D FBP-type reconstruction methods for helical CB-CT. However, it is difficult to do a quantitative comparison. Furthermore, we observe that interrupted illumination does not produce visible artifacts. The number of events that a voxel enters the cone for the images shown in Fig. 15 are displayed in Fig. 16.

\section{DISCUSSION}

Two important points have to be addressed to delimitate the $n$-PI-FBP method from other helical FBP approaches: the so-called long object problem and the relation to the helical Feldkamp method:

An important problem for helical scanning is the so-called long object problem. If the data acquisition and the reconstruction should only be applied to a limited part of a long object that exceeds the scanned region, we have to address truncation problems at the beginning and at the end of the helix. Tam has proposed to perform an additional circular scan at the beginning and at the end of the scanned region. As it becomes obvious from the discussion of the Radon plane segmentation, the same solution can be applied to the $n$-PI-method. The long object problem does not exist for Danielsson's PI-FBP algorithm and for the $n$-PI-FBP algorithm.

In the following, we investigate the obvious question: can $n$ be even? The answer has several facets. After an analysis of two theoretical aspects, we briefly review a group of approximative methods sometimes called helical Feldkamp algorithm.

The consideration of the PI-sufficiency condition from Danielsson leads to the question of whether a 2-PI-sufficiency condition makes sense. Independent of the answer, we can easily prove that such a condition could not be fulfilled with a helical source trajectory. The source position would have to be identical for a sunrise and a sunset event. This will never happen on a helical trajectory of the source.

The key point of a Tam-style triangulation of Radon planes is the fact that the individual segments do not overlap. The possible positions of the source at the borders can either be on opposite positions leading to a odd $n$-PI-case or at the same position, a requirement not fulfilled for helical source trajectories.

The adaptation of the Feldkamp algorithm to helical acquisition trajectories leads to another group of approximative reconstruction algorithm. Such an algorithm has been described by Wang et al. [13]. The basic difference of the Wang method and the Feldkamp method is that during the backprojection, each object point gets only a contribution from projections with a angular span of $2 \pi$, as seen in a projection to the $x y$-plane. This 

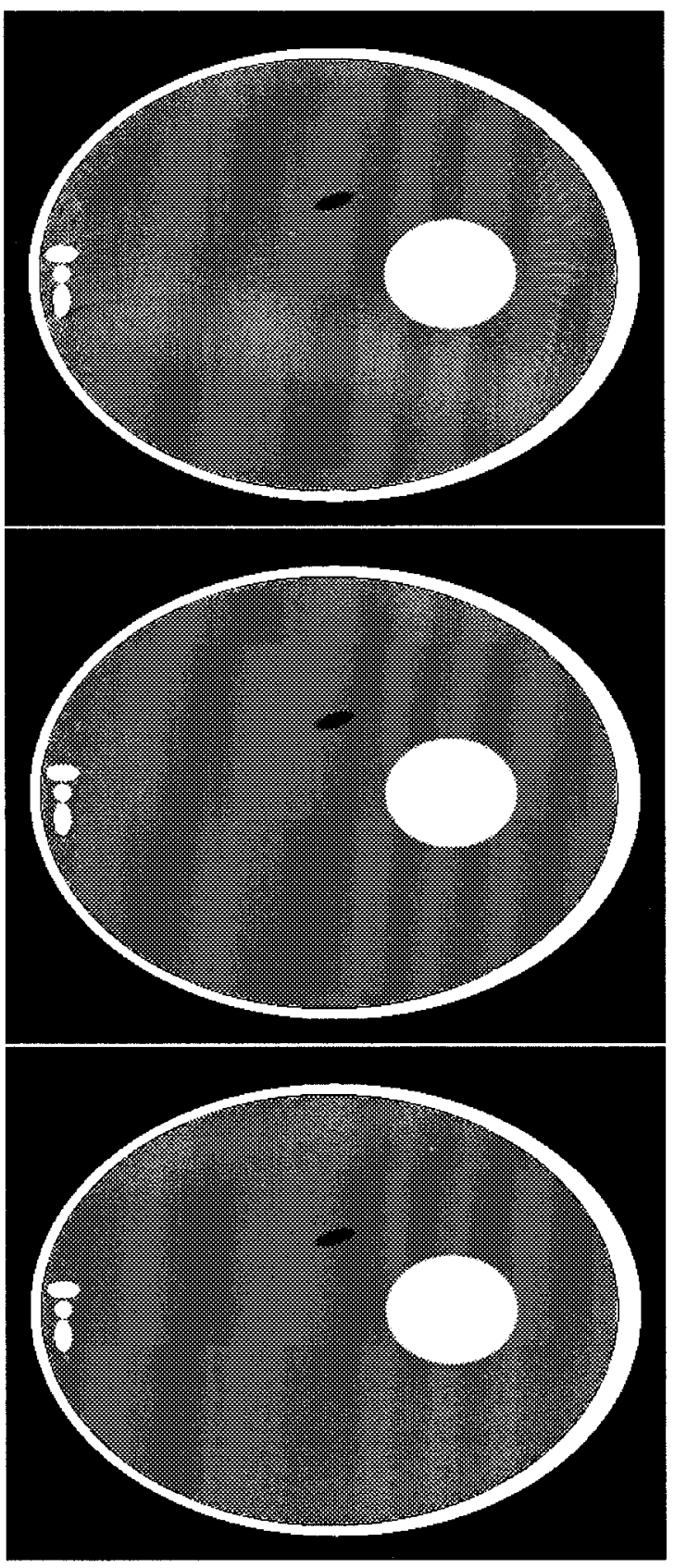

Fig. 13. Axial slice through the reconstructed Shepp-Logan phantom for the 16 array detector using the $n$-PI-FBP-method in 1-PI-mode (top), 3-PI-mode (middle), and 5-PI-mode (bottom). Level: $20 \mathrm{HU}$; window: $10 \mathrm{HU}$.

modification is necessary to remove redundant data of the helical acquisition proposed by Wang. Neglecting the cone-angle or the moving $z$-position, each object point gets contribution over a span of $2 \pi$. An easy extension can be made to $4 \pi, 6 \pi, \cdots$ and is discussed, e.g., by Schaller [12].

Fig. 17 shows a Radon plane containing the rotation axis for the $2 \pi$-case. Three source positions $S_{0}, S_{1}, S_{2}$ and the related intersections of the cone with the plane are shown. The source positions on the opposite side are not drawn and are not discussed, because they behave symmetrically. The object cylinder with radius $r$ defines the maximal pitch, because all points on the object cylinder must be illuminated over an angular span of $2 \pi$. Point $A$ for example is illuminated starting at source posi-
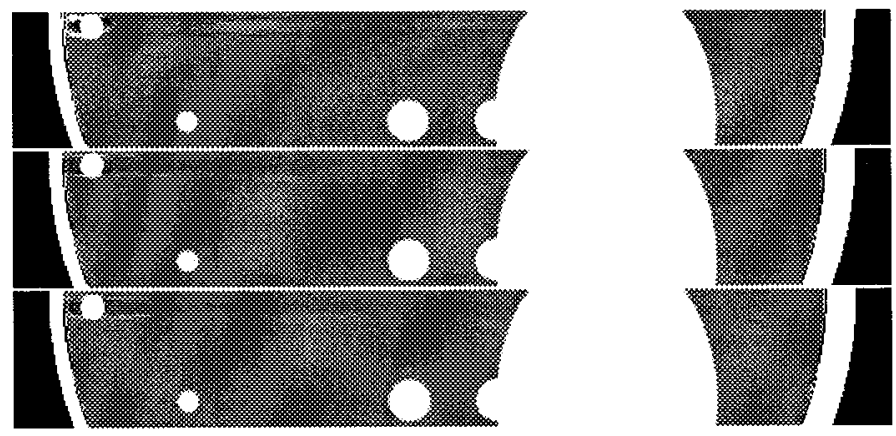

Fig. 14. Mid-sagittal slice through the reconstructed Shepp-Logan phantom for the 64 array detector using the $n$-PI-FBP-method in 1-PI-mode (top), 3-PI-mode (middle), and 5-PI-mode (bottom). Level: $20 \mathrm{HU}$; window: $10 \mathrm{HU}$.

tion $S_{0}$ until position $S_{1}$. For the drawn Radon plane, all object points in the rectangle $A, B, E, D$ get a contribution during the backprojection from source position $S_{1}$ only. A proper condition in the backprojection of the Wang algorithm takes care that no contribution from other projections $\left(S_{0}\right.$ and $\left.S_{2}\right)$ are used. The areas outside of this rectangle do not get a contribution from source position $S_{1}$. The two triangles $A, C, D$ and $B, E, F$ are exposed from source position $S_{1}$, but the acquired information is not used to reconstruct the object points there. This relation shows that if we acquire sufficient data to cover the complete Radon plane, we will get redundancies. These redundancies are removed during the conditional backprojection of the Wang algorithm. They do not allow a simple adaptation of the Tam Radon plane segmentation. For medical applications, these redundancies lead to a dose utilization problem, because a substantial part of the exposure is not properly used. For a practical geometry $(R=500 \mathrm{~mm}, r=250 \mathrm{~mm})$, the area of the unnecessary exposed regions $(A, C, D$ and $B, E, F)$ is $50 \%$ of the total exposed area for the Radon plane shown in Fig. 17. This wasting of dose becomes even more obvious, if the maximum possible pitch, as published, e.g., in Schaller [12], is calculated for the two scanner geometries used in our simulation: For Det16, it is $7.7 \mathrm{~mm}$, whereas for Det64 it is $32 \mathrm{~mm}$. These pitches are still smaller than needed for a 3-PI-method $(8.3 \mathrm{~mm}$, $33 \mathrm{~mm}$ ), whereas Wang's algorithm allows only for a 2-PI-reconstruction.

\section{CONCLUSION}

We have defined a new class of helical CB-CT acquisition geometries. We have shown how exact and quasiexact reconstruction methods based on Grangeat's result and a Radon plane triangulation can be adapted to fit them. The triangulation and the redundancy of the acquisition windows have been discussed. It has been shown how the Defrise-Kudo $M$-function can be defined and used in exact reconstruction methods. We have shown that the acquisition geometry fulfills Danielsson's sufficiency condition. We have analyzed the noncontinuous illumination for high PI-modes and shown that the acquired data can be combined into a continuous angular illumination using complementary rays. Moreover, we have demonstrated with numerical simulations that the image quality of the $n$-PI-FBP method is suitable for medical applications. The unbeatable scan efficiency of the PI-window measured as scanned volume per rotation and the 

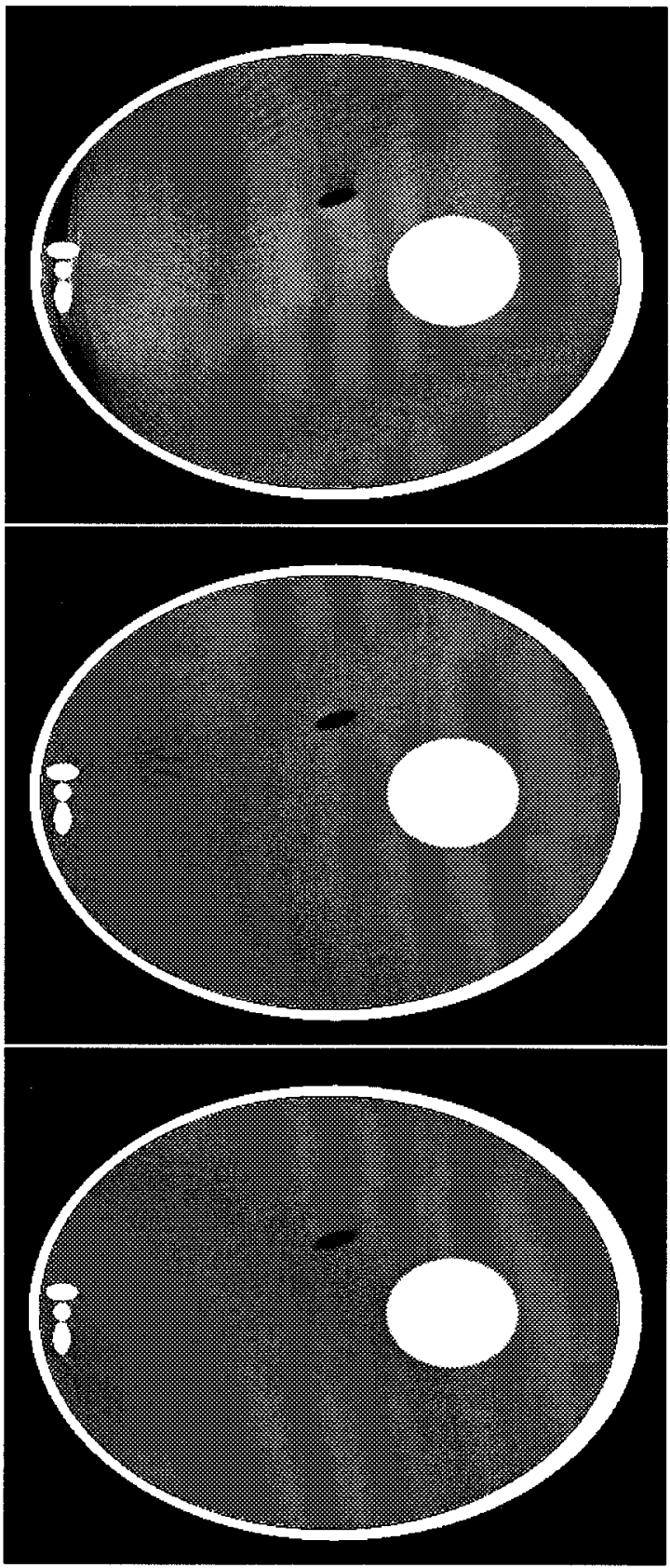

Fig. 15. Axial slice through the reconstructed Shepp-Logan phantom for the 64 array detector using the $n$-PI-FBP-method in 1-PI-mode (top), 3-PI-mode (middle), and 5-PI-mode (bottom). Level: $20 \mathrm{HU}$; window: $10 \mathrm{HU}$.

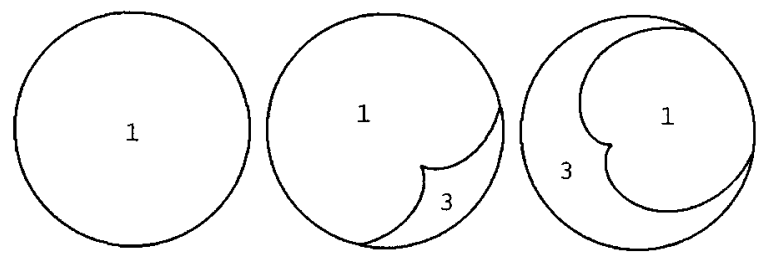

Fig. 16. Contour plot of the number of events that an object point comes into the cone related to the 1-PI-(left), 3-PI-(middle), and 5-PI-modes (right) for the reconstructions that are shown in Fig. 15. The circle indicates the field of view.

better image quality when using higher PI-modes can be combined in a medical CT scanner. Depending on the actual applica-

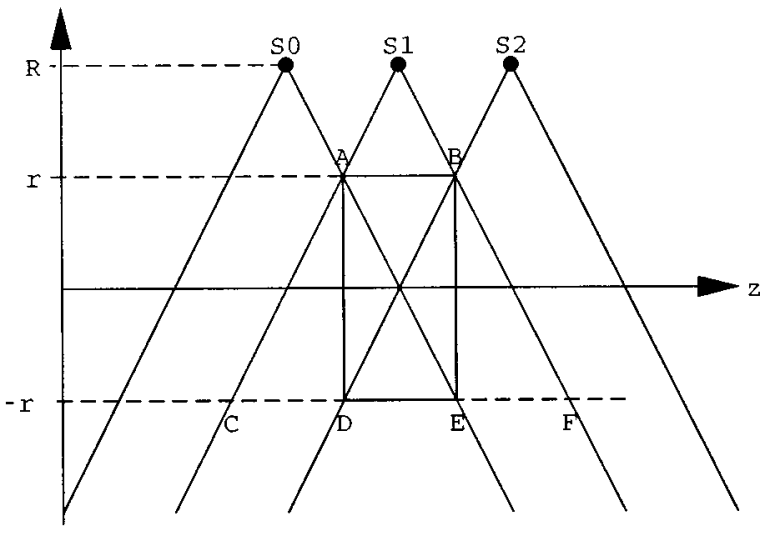

Fig. 17. Illustration of the helical Feldkamp algorithm as published by Wang. Three source positions $S_{0}, S_{1}$, and $S_{2}$ and the object cylinder with radius $r$ is shown. The points $A, \cdots, F$ are referred in the explanation to analyze the dose utilization.

tion, the PI-mode can be used to tune the system with respect to image quality and scan speed. The pitch parameter, which can have any value in conventional helical CT scanning, has now been replaced by a discrete equivalent, the PI-mode.

A number of technical and image-quality-related advantages have been identified. Especially, the 3-PI-FBP method seems to be an excellent candidate for medical CB-CT, providing high image quality and fast scanning.

\section{APPENDIX I}

\section{A. Triangulation of the Radon Planes}

We examine the intersection of an arbitrary Radon plane $\Pi(l, \vec{\xi})$ with the source cylinder $x^{2}+y^{2}=R^{2}$ and the helix vertex $\vec{a}(\lambda)=(R \cos \lambda, R \sin \lambda, h \lambda)$ for $\lambda \in \Lambda$. The intersection of $\Pi(l, \vec{\xi})$ with the source cylinder defines an ellipse $E$.

Let us define coordinates $(\vec{\zeta}, \vec{\eta}, \vec{\xi})$ such that $\vec{\zeta}=\vec{\xi} \times \vec{z}$ and $\vec{\eta}=\vec{\xi} \times \vec{\zeta}$. If $E$ exists, it will be in the $(\vec{\zeta}, \vec{\eta})$-plane with the long main axis in $\vec{\eta}$ and the short axis in $\vec{\zeta}$. The plane $\Pi(l, \vec{\xi})$ intersects with the source path on $E$ at some points

$$
\vec{a}\left(\lambda_{i}\right) \quad \text { for } i=1, \cdots, n_{0}(\vec{\xi}, l) .
$$

The number of intersections $n_{0}$ is odd or zero. ${ }^{6}$

The numbering is done in such a way that the intersections are ordered in $\lambda\left(\lambda_{i}<\lambda_{i+1}\right)$. The related $\vec{\eta}$-coordinates are monotone $\vec{\eta}_{i} \geq \vec{\eta}_{i+1}$ or $\vec{\eta}_{i} \leq \vec{\eta}_{i+1}$. We discuss only the first case and treat the second by virtually inverting the $\vec{\eta}$-axis. All $n$-PI-windows, including the Tam-Danielsson window, will deliver redundant data for some Radon planes, a problem that is discussed later in this appendix. We use the term quasi-nonredundant to exclude any additional redundancy.

We distinguish five cases, as follows:

- Case 1: $n_{0}=0$.

- Case 2: $n_{0}=1$.

- Case 3: $n_{0}=3, n=1$ and $n_{0}=3, n \geq 3$.

${ }^{6} \mathrm{We}$ will make some statements about intersections of a plane with a helix. They can easily be verified in a plot of the $\vec{z} \vec{\xi}$ projection, as shown in Fig. 18 . The plane reduces to a line, and the helix becomes a sine with an arbitrary phase. The sign of the related cosine shows the side of the helix with respect to the $\vec{\zeta}$-axis. 


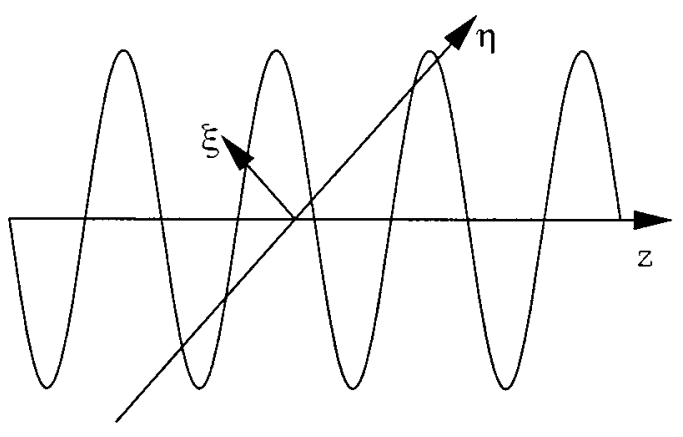

Fig. 18. Vertex path $\Lambda$ and a Radon plane with normal $\xi$ seen perpendicular to the $z$-axis and to $\xi$.

- Case 4: $n_{0} \geq 5$ and all successive vertices are on opposite sides of $\vec{\eta}$

$$
\left[\vec{a}\left(\lambda_{i}\right) \cdot \vec{\zeta}\right]\left[\vec{a}\left(\lambda_{i+1}\right) \cdot \vec{\zeta}\right]<0 \quad \text { for } i=1, \cdots, n_{0}-1
$$

- Case 5: $n_{0} \geq 5$ and all but the last or first two successive vertices are on opposite sides of $\vec{\eta}$. Opposite sides are defined as in (36).

Case $1\left(n_{0}=0\right)$ : The Radon plane does not intersect the object cylinder that includes the support of the object function. The related Radon value is known to be zero.

Case $2\left(n_{0}=1\right)$ : The Radon plane $\Pi(l, \vec{\xi})$ is completely and nonredundantly covered by a single source position. The related $M$-function is

$$
M(l, \vec{\xi}, \lambda)=1 \quad \text { for } n_{0}=1 .
$$

Case 3 ( $n_{0}=3, n=1$, illustrated in Fig. 19): This is discussed in detail for the PI-window in [7] and [27]. The plane is completely and quasi-nonredundantly covered by three segments, whereas two segments are redundant. Even if the number of source positions is greater than 1 , the plane is not overrepresented in the Radon domain and

$$
M(l, \vec{\xi}, \lambda)=1 \quad \text { for } n_{0}=3, n=1 .
$$

The extension of this case to the $n$-PI-window, for $n \geq 3$, must be treated in a different way. Because the maximum $\lambda$-span for each possible pair of intersections does not exceed $3 \pi$, the segments seen by each source position are not truncated by a detector boundary. If a segment is not truncated by a detector boundary, it illuminates the Radon plane completely. As a result, the Radon plane is completely and homogeneously covered three times. Because the projections are not truncated, exact reconstruction methods can easily treat this case with a proper $M$-function, which is

$$
M(l, \vec{\xi}, \lambda)=1 / 3 \quad \text { for } n_{0}=3, n \geq 3 .
$$

Case $4\left[n_{0} \geq 5\right.$ and all successive intersections $\vec{a}\left(\lambda_{i}\right)$ are on opposite sides of the $\vec{\eta}$-axis, the long axis of $E$, for $i=$ $1, \cdots, n_{0}$, illustrated in Fig. 5]: For each pair of intersections $\vec{a}\left(\lambda_{i}\right)$ and $\vec{a}\left(\lambda_{i+m}\right)$ for $m \in N, i \geq 1$ and $i+m \leq n_{0}$, we have

$$
(m-1) \pi \leq \lambda_{i+m}-\lambda_{i}<(m+1) \pi .
$$
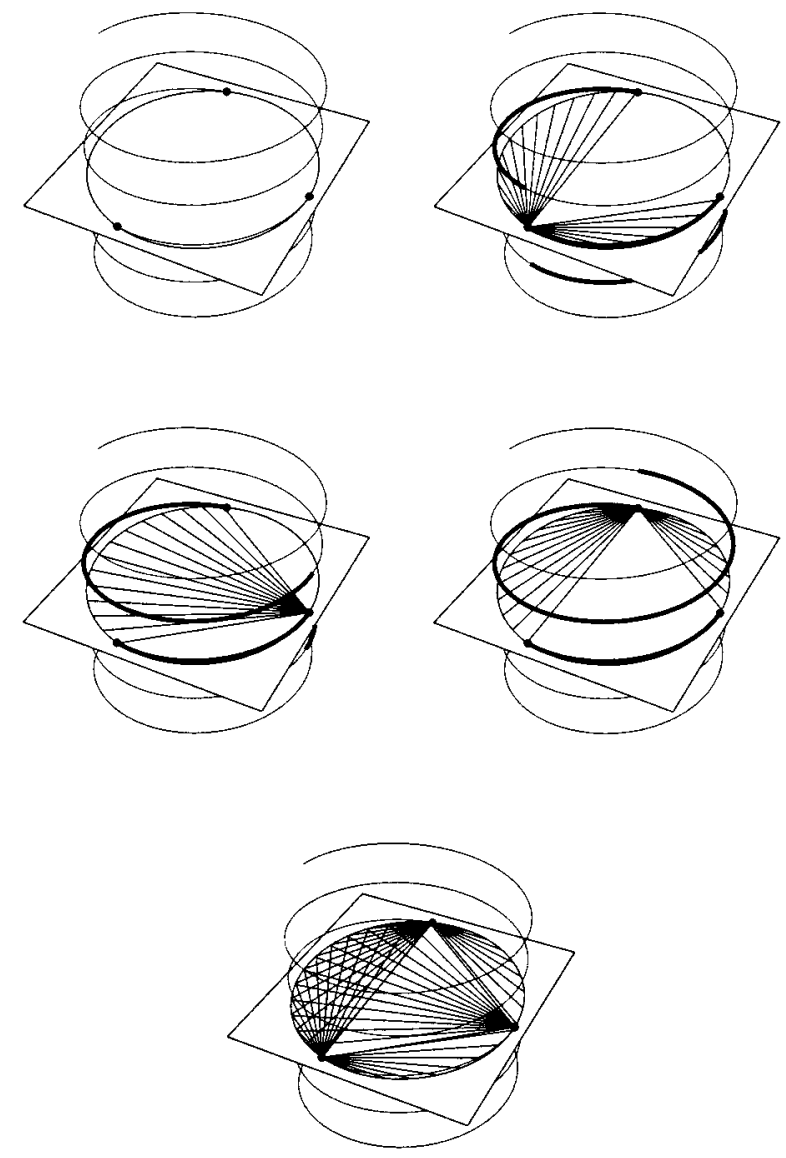

Fig. 19. A Radon plane intersecting the helix (top left) and the segments seen by the different vertex positions for the 1-PI-mode (top right to middle right) and the total coverage of the plane. The measured part of the Radon plane for each vertex position is hatched, and the lower and upper detector boundary is drawn thick, assuming a fan-angle of $180^{\circ}$. A significant fraction of the plane is covered redundantly.

We like to stress the point that this relation is only valid if we assume that successive vertices are on opposite sides of $E$. The relation (40) defines a $\lambda$-range for successive intersections. To identify the illuminated sector of a Radon plane of a given source vertex $\vec{a}\left(\lambda_{i}\right)$, we have to identify the related $n$-PI-lines that connect the source vertex with the lower or upper detector boundary. We have defined the angular range of the $n$-PI-window in (6) and (7). This range can be mapped to (40) as follows.

- Mapping $1\left(m_{l}=-n\right)$ and $\left(m_{u}=n\right)$ leads to the following solutions for source vertex $\vec{a}\left(\lambda_{i}\right)$.

a) If ( $i-n \geq 1)$, the line connecting $\vec{a}\left(\lambda_{i}\right)$ and $\vec{a}\left(\lambda_{i-n}\right)$ is the lower $n$-PI-line. If $(i-n<1)$, the complete region from the upper $n$-PI-line toward the lower part of $E$ is illuminated from source vertex $\vec{a}\left(\lambda_{i}\right)$. This is because the segment is not truncated toward the lower part of $E$ by a detector boundary.

b) If ( $\left.i+n \leq n_{0}\right)$, the line connecting $\vec{a}\left(\lambda_{i}\right)$ and $\vec{a}\left(\lambda_{i+n}\right)$ is the upper $n$-PI-line. If $\left(i+n>n_{0}\right)$, the complete region from the lower $n$-PI-line toward the upper part of $E$ is illuminated from source vertex 
$\vec{a}\left(\lambda_{i}\right)$. Because $n$ is odd, the related $n$-PI-lines connect points on opposite sides of $E$.

- Mapping $2\left(m_{l 1}=1-n\right)$ or $\left(m_{l 2}=-1-n\right)$ or $\left(m_{u 1}=n-1\right)$ or $\left(m_{u 2}=n+1\right)$. This mapping will define additional segments of illumination for a given source vertex. The related $n$-PI-lines connect vertex points on the same side of the ellipse $E$. Because these areas are also illuminated from other vertex points, this solutions will result in a redundancy that cannot be treated with a global $M$-function with respect to $\Pi(l, \vec{\xi})$.

We conclude that all connections $\vec{a}\left(\lambda_{i}\right) \rightarrow \vec{a}\left(\lambda_{i+n}\right)$ are $n$-PIlines.

Some remarks on the redundancies are in order. The redundancy are present in all $n$-PI-windows. They have been discovered for the first time by Kudo et al. [27] for the $\pi$-case. Fig. 19 shows an example for the PI-case.

The problem can be treated for the exact reconstruction methods by a proper integration interval over $t$. For a real CB-CT scanner, the problem is not critical because the redundant areas are most often at the borders of the ellipse $E$. These areas are often outside of the cone-beam in fan direction. In the PI-FBP method and in the $n$-PI-FBP method, this redundancy is not addressed.

We can now analyze the illumination of a Radon plane for Case 4. We start to investigate the illumination of an arbitrary segment that is defined by two existing (real) $n$-PI-lines, the connections of points $\vec{a}\left(\lambda_{i}\right) \rightarrow \vec{a}\left(\lambda_{i-n}\right)$ and $\vec{a}\left(\lambda_{i}\right) \rightarrow \vec{a}\left(\lambda_{i+n}\right)$; see Fig. 20. Let us take an arbitrary point $P_{1}$ within this sector. The point $P_{1}$ is illuminated by a certain amount of vertices from the right-hand side of the ellipse $E$. The first and last vertex of this range has index $j$ and $k$. From the left-hand side of $E$, point $P_{1}$ is illuminated from $\vec{a}\left(\lambda_{k-n+2}\right)$ to $\vec{a}\left(\lambda_{j+n-2}\right)$. Counting all illumination events, we get

$$
\left(\frac{k-j}{2}+1\right)+\left(\frac{(j+n-2)-(k-n+2)}{2}+1\right)=n .
$$

If a point $P_{2}$ is not illuminated from the right-hand side, there are two vertices on the left-hand side $j-n$ and $k+n$ for which the upper $n$-PI-line of $\vec{a}\left(\lambda_{j-n}\right)$ is below $P_{2}$ and the lower $n$-PI-line of $\vec{a}\left(\lambda_{k+n}\right)$ above $P_{2}$. Counting the number of illuminations from the left-hand side of $E$, we get

$$
\frac{(k+n)-(j-n)}{2}+1 .
$$

With $k=j+2$, we have again $n$-illuminations.

If we analyze the situation close to the top or bottom of the ellipse $E$, we can treat the nonexisting vertices $\vec{a}\left(\lambda_{i}\right)$ for $i<1$ or $i>n_{0}$ as a complete coverage of the remaining part of $E$. Similar arguments as used for the inner part can be used to prove that the number of illuminations is always $n$ for $n_{0}>n$.

Knowing the $\lambda$-index relations of $n$-PI-line points, we can easily see that for the cases $n_{0} \leq n$, no $n$-PI-lines are present. All $n_{0}$-vertices will illuminate the ellipse completely.

Case 5 ( $n_{0} \geq 5$ and all but the last or first two successive vertices are on opposite sides of $\vec{\eta}$, illustrated in Fig. 21): This case is identical to Case 4 with the exception that the first two or

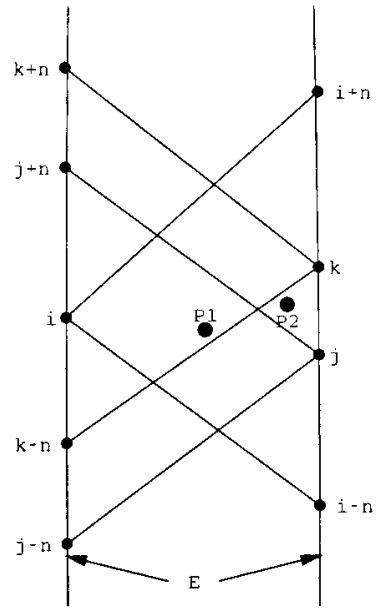

Fig. 20. A part of a long ellipse, two object points $P_{1}$ and $P_{2}$, and some intersection points of the Radon plane with the vertex path. The $n$-PI-lines connecting source positions $i$ and $i+n$ are also shown.

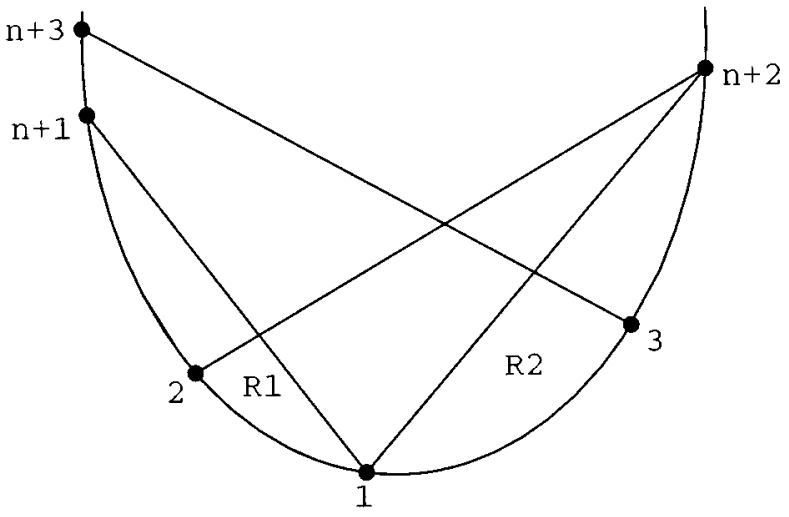

Fig. 21. Triangulation of a Radon plane in the $n$-PI-window, where the first two vertices are on the same side of the main axis. The intersection points are labeled according to their occurrence in time. The normal $n$-PI-lines connecting vertex positions 1 with $1+n$ and the extra $n$-PI-line connecting vertex position 1 with $2+n$ are also shown.

the last two intersections are on the same side of the ellipse $E$. Due to the inherent symmetry of the problem, we discuss only the case in which the first two vertices are at the left-hand side of the ellipse. The other cases can be treated by inverting the $\vec{\eta}$-axis or the $\vec{\zeta}$-axis or both. The vertex point $\vec{a}\left(\lambda_{1}\right)$ does not fit in our description so far. Because the next vertex is on the same side, (40) must be adapted for vertex $\vec{a}\left(\lambda_{1}\right)$ to

$$
(m-1) \pi \leq \lambda_{m}-\lambda_{1}<m \pi \text {. }
$$

The possible mappings of (6) and (43) show again two solutions for $m=n$ and $m=n+1$. Both solutions produce $n$-PI-lines if the related $n$-PI-line partner exists $\left(m \leq n_{0}\right)$. The area above the $n$-PI-line connecting $\vec{a}\left(\lambda_{3}\right)$ and $\vec{a}\left(\lambda_{3+n}\right)$ cannot be analyzed with the results from Case 4 . The situation is illustrated in Fig. 21. As outlined in the description of the possible mappings, vertex $\vec{a}\left(\lambda_{1}\right)$ has $n$-PI-lines to vertices $\vec{a}\left(\lambda_{n+1}\right)$ and $\vec{a}\left(\lambda_{n+2}\right)$. As in the other cases, we can simply count the number of illuminations in the remaining areas. The result shows that all regions except region $R_{1}$ and $R_{2}$ are covered $n$-times. In region 
a

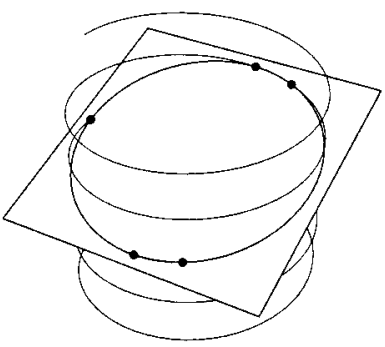

$\mathrm{b}$

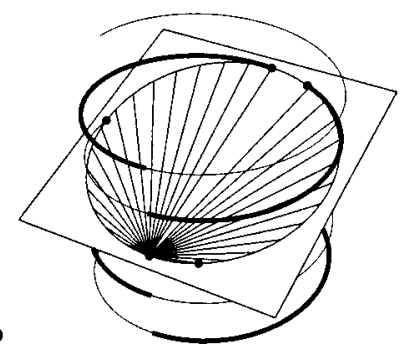

$\mathrm{d}$

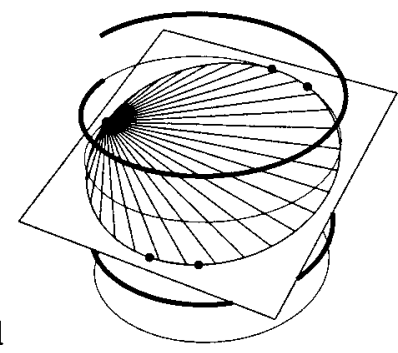

$\mathrm{C}$
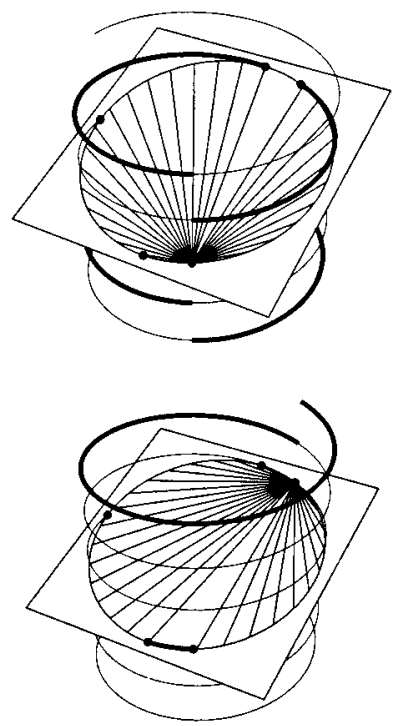

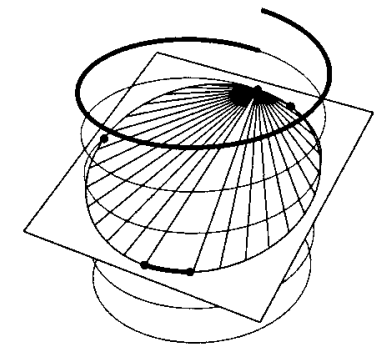

Fig. 22. A Radon plane intersecting the helix (a) and the segments seen by the different vertex positions, indicated as hatched areas, for the 3-PI-window (b)-(f), which are ordered according to their occurrence in time. In (b)-(f), the lower and upper detector boundary is drawn thick, assuming a fan-angle of $180^{\circ}$. Note that the right half of the fan (as seen from the source) of (b) and (e) as well as the right halfs of the fans of (d) and (f) fill the whole plane. A significant fraction of the plane is covered redundantly by more than a factor of 3 , i.e. the left halfs of the fans in (b), (d), (e), and (f) contain redundant data.

$R_{1}$, we have $n+1$-illuminations, and in region $R_{2}$, we observe $n+2$-illuminations. An example of this case is shown in Fig. 22. As for the other redundancies, these cases are rare and become most often noneffective due to the limited object radius.

We summarize the results of the discussion with a compact description of the $M$-function that represents the inverse of the number of complete illuminations of the Radon plane. Ignoring the inherent redundancy of the $n$-PI-acquisition geometry, the proper $M$-function is

$$
M_{n}(l, \vec{\xi}, \lambda)= \begin{cases}\frac{1}{n_{0}(\lambda, l)}, & n_{0}(\lambda, l)<n \\ \frac{1}{n}, & \text { otherwise }\end{cases}
$$

\section{B. Proof that the Rebinned Detector is Rectangular}

Without loss of generality, we consider the case $h=1$ and $R=1$. Fig. 23 shows a top view onto the helix. The actual vertex $a$ is at angle $\lambda$, and we consider a ray with fan-angle $\gamma$

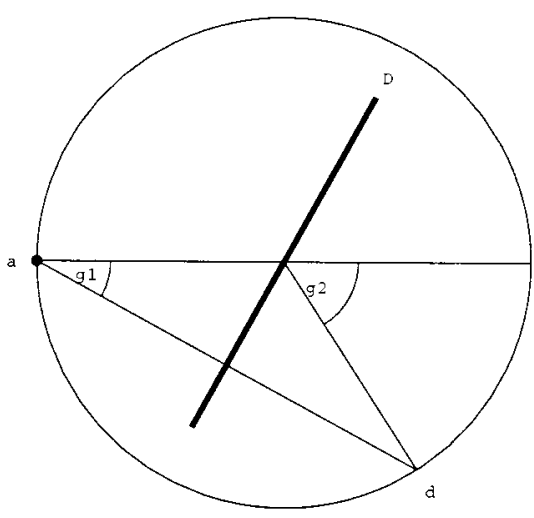

Fig. 23. Top view onto the helix with a vertex $a$, a certain ray with fan-angle $\gamma$, hitting the detector boundary at $d$, and the virtual detector $D$ to which this ray contributes.

that hits the upper boundary of the detector. According to (6) and (7), this boundary is at

$$
d=(\cos (\lambda+\pi+2 \gamma), \sin (\lambda+\pi+2 \gamma) .
$$

This ray contributes to the virtual detector $D$, as indicated in Fig. 23. As the triangle spanned by $a$, the rotation axis, and $d$ is isosceles, the $z$-position of the intersection of the ray with the virtual detector $D$ is

$$
\begin{aligned}
z & =\left(d_{z}+a_{z}\right) / 2 \\
& =\lambda+\gamma+n \pi / 2 \\
& =\theta+n \pi / 2,
\end{aligned}
$$

where $\theta$ denotes the projection angle of the virtual detector (30). Therefore, all rays contributing to the projection angle $\theta$ that hit the upper boundary of the physical detector, intersect the virtual detector at $z=n \pi / 2$, which is then a rectangle.

\section{The Minimal Radius of an Object Point that Comes into the Cone More than Once}

The phenomenon that an object point enters the cone several times occurs only for object points that have a certain minimum distance $\varrho$ from the rotation axis. This minimum distance can be calculated in the following way. Consider a fixed, virtual detector and an object point $\vec{r}$ lying on the lower $n$-PI surface; i.e., an object point that is projected onto the lower boundary of the virtual detector (see Fig. 24). For this object point, we determine its velocity vector $\vec{v}$ and the local normal $\vec{n}$ of the $n$-PI surface. If

$$
\vec{n} \cdot \vec{v}<0,
$$

the object point crosses the $n$-PI-surface from inside to outside.

First, it should be noticed that in the situation, as shown in Fig. 24, with the object point moving counterclockwise upward, this phenomenon can only occur if the $x$-coordinate of $\vec{r}$ is positive.

The $v$-component of the projection of an arbitrary object point $(x, y, z)$ onto the virtual detector is given in (34) for properly scaled coordinates, as described in Section VII. The lower $n$-PIsurface is defined as the set of points that have $v(x, y, z)=$ 

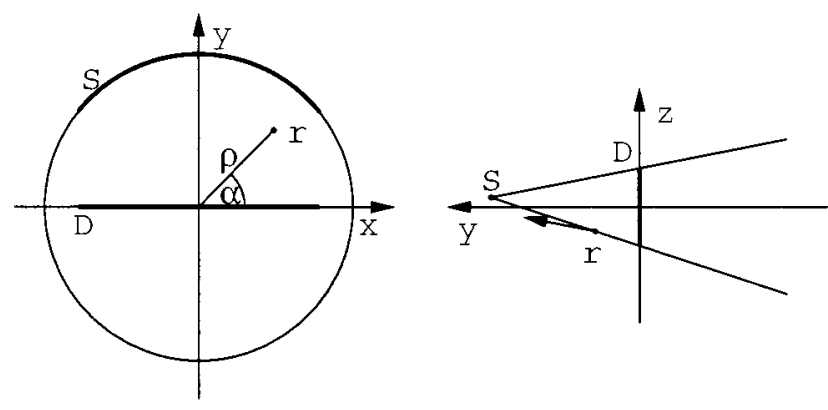

Fig. 24. Schematic view of an object point $r$ with coordinates $(\rho, \alpha)$ moving in the rebinned source-detector arrangement. The detector $D$ is parallel to the $x$-axis, and the extended source is located in the upper half of the picture. The object point moves counterclockwise upward. Left: Top view. Right: View in the plane containing $r$ perpendicular to the $x$-axis. The direction of movement of the object point is also indicated as well as the projection of the $y$ - and $z$-axis onto this plane.

$-n \pi / 2$; see (48). The normal of the $n$-PI-surface is therefore proportional to

$$
\begin{aligned}
\vec{n} & \propto \nabla v(x, y, z) \\
= & \left(\begin{array}{c}
-\frac{y\left(\sqrt{1-x^{2}}-y-x z+x \arcsin (x)\right)}{\sqrt{1-x^{2}}\left(\sqrt{1-x^{2}}-y\right)^{2}} \\
\frac{\sqrt{1-x^{2}}(z-\arcsin (x))}{\left(\sqrt{1-x^{2}}-y\right)^{2}} \\
\frac{\sqrt{1-x^{2}}}{\sqrt{1-x^{2}}-y}
\end{array}\right) .
\end{aligned}
$$

Now, we calculate the velocity vector of the object point $\vec{r}=$ $(x, y, z)$

$$
\frac{\partial}{\partial t} \vec{r}=(-y, x, 1)
$$

using the fact that the object point moves on a helical path upward with a speed in $z$-direction equal to the pitch.

As we consider a point lying on the lower $n$-PI-surface, $v(x, y, z)=-n \pi / 2$. By solving (34) for $z$, we get a relation between $z$ and $x, y$

$$
z=-\frac{n \pi}{2}+\frac{n \pi y+2 y \arcsin (x)}{2 \sqrt{1-x^{2}}} .
$$

Now, we can evaluate (53), as shown at the bottom of the page. As we are looking for the minimum radius where an object point moves out several times, we set now $x=\varrho \cos (\alpha), y=$ $\varrho \sin (\alpha)$, and we look for zeros of

$$
g(\varrho, \alpha)=\vec{n} \cdot \frac{\partial}{\partial t} \vec{r}
$$

resulting in

$$
\begin{aligned}
0= & 2 \sqrt{1-\varrho^{2} \cos ^{2}(\alpha)}\left(\varrho^{2}\left(\cos ^{2}(\alpha)-\sin ^{2}(\alpha)\right)-1\right) \\
& -\varrho\left(n \pi+2 \arcsin (\varrho \cos (\alpha)) \cos (\alpha)\left(\varrho^{2}-1\right) .\right.
\end{aligned}
$$

TABLE III

Minimum Distance of AN OBJeCt POINT TO THE Rotation AXIS WHERE IT COMES INTO THE CONE MORE THAN ONCE FOR DIFFERENT PI-WINDOWS

\begin{tabular}{c||c|c|c|c}
\hline$n$ & 1 & 3 & 5 & 7 \\
\hline \hline$\varrho_{0}$ & 1 & 0.199 & 0.124 & 0.090 \\
\hline
\end{tabular}

TABLE IV

DETECTOR Utilization of a Rectangular, Focus CENTERED Detector FOR DIFFERENT PI-WINDOWS

\begin{tabular}{c||c|c|c|c}
\hline PI-Mode $n$ & 1 & 3 & 5 & 7 \\
\hline \hline$F_{n} / G_{n}[\%]$ & 73.3 & 85.7 & 88.7 & 90.0 \\
\hline
\end{tabular}

The zeros of $g$ have been determined numerically, and the minimal values for $\varrho$ are given in Table III. It turned out that the minimum is always reached for $\alpha=\pi / 2$. It further turned out that at these values, the object point really crosses the $n$-PI-surface.

\section{Detector Utilization}

For technical reasons, it is likely that a real detector would have a rectangular shape on a cylinder centered around the focal spot of the X-ray tube. If we project the lower and upper border of the $n$-PI-window (6), (7) onto this cylinder relative to the source position and parameterize it with the fan-angle $\gamma$, we get

$$
\begin{aligned}
& \tilde{U}_{n}(\gamma)=\frac{D h\left(\frac{\gamma}{\pi}+\frac{n}{2}\right)}{2 R \cos \gamma}, \\
& \tilde{L}_{n}(\gamma)=-\tilde{U}_{n}(-\gamma),
\end{aligned}
$$

with $D$ being the source-detector distance. The detector area required for a maximal fan-angle of $\pm \gamma_{\max }$ is

$$
\begin{aligned}
F_{n}\left(\gamma_{\max }\right) & =2 D \int_{0}^{\gamma_{\max }} d \gamma\left(\tilde{U}_{n}(\gamma)-\tilde{L}_{n}(\gamma)\right. \\
& =\frac{n D^{2} h}{R} \ln \tan \left(\frac{\gamma_{\max }}{2}+\frac{\pi}{4}\right) .
\end{aligned}
$$

A proper rectangular detector sufficient to acquire all required data has an area of

$$
\begin{aligned}
G_{n}\left(\gamma_{\max }\right) & =\left(D 2 \gamma_{\max }\right) 2 \tilde{U}_{n}\left(\gamma_{\max }\right) \\
& =\frac{D^{2} h \gamma_{\max }\left(2 \gamma_{\max }+n \pi\right)}{R \pi \cos \gamma_{\max }} .
\end{aligned}
$$

For a fan-angle of $\gamma_{\max }= \pm 25^{\circ}$, we get a detector utilization as given in Table IV.

\section{ACKNOWLEDGMENT}

The authors would like to thank P.-E. Danielsson and his research group at the University of Linköping, Sweden, especially $\mathrm{H}$. Turbell and M. Magnusson-Seger, for the advice on the PI-method and for numerous fruitful discussions.

$$
\vec{n} \cdot \frac{\partial}{\partial t} \vec{r} \propto \frac{2 \sqrt{1-x^{2}}\left(x^{2}-y^{2}-1\right)-x\left(x^{2}+y^{2}-1\right)(n \pi+2 \arcsin (x))}{2\left(x^{2}-1\right)\left(\sqrt{1-x^{2}}-y\right)}
$$




\section{REFERENCES}

[1] K. Taguchi and H. Aradate, "Algorithm for image reconstruction in multi-slice helical CT," Med. Phys., vol. 25, no. 4, pp. 550-561, 1998.

[2] F. Noo, M. Defrise, and R. Clackdoyle, "Single slice rebinning method for helical cone-beam CT," Phys. Med. Biol., vol. 44, pp. 561-570, 1999.

[3] H. Hu, "Multi-slice helical CT: Scan and reconstruction," Med. Phys., vol. 26, no. 1, pp. 5-18, 1999.

[4] G. Larson, C. C. Ruth, and C. R. Crawford, "Nutating slice CT image reconstruction apparatus and method," U.S. Patent 5,802,134, 1998.

[5] E. M. Bailey, C. R. Crawford, A. I. Greenberg, and C. C. Ruth, "Parallel processing architecture for computed tomography scanning system using nonparallel slices," U.S. Patent 5,887,047, 1999.

[6] K. C. Tam, "Three-dimensional computerized tomography scanning method and system for large objects with smaller area detectors," U.S. Patent 5,390,112, 1995.

[7] K. C. Tam, S. Samarasekera, and F. Sauer, "Exact cone beam CT with a spiral scan," Phys. Med. Biol., vol. 43, pp. 1015-1024, 1998.

[8] P. E. Danielsson, P. Edholm, J. Eriksson, and M. Magnusson-Seger, “Toward exact 3D-reconstruction for helical cone-beam scanning of long objects," in Proc. 3D'97 Conf., Nemacolin, PA, pp. 141-144.

[9] J. Eriksson, "Problems and experiments in cone-beam tomography," Tech. Rep. Lic. thesis 710, Linköping Univ., Sweden, 1998.

[10] H. Turbell, "Three-dimensional image reconstruction in circular and helical computed tomography,” Tech. Rep. Lic. thesis no. 760, Linköping Univ., 1999.

[11] C. Jacobsen, "Fourier methods in 3D-reconstruction from cone-beam data," Tech. Rep. Ph.D. thesis 427, Linköping Univ., Sweden, 1996.

[12] S. Schaller, "Practical image reconstruction for cone-beam computed tomography," Tech. Rep. Ph.D. thesis, Univ. Erlangen, Germany, 1998

[13] G. Wang, T. H. Lin, P. C. Cheng, and D. M. Shinozaki, "A general cone-beam reconstruction algorithm," IEEE Trans. Med. Imag., vol. 12, pp. 486-496, Mar. 1993.

[14] G. Wang, T. H. Lin, and P. C. Cheng, "Error analysis on a general Feldkamp's cone-beam computed tomography algorithm," Scanning, vol. 17, pp. 361-370, 1995.

[15] M. D. Silver, "High helical pitch cone-beam computed tomography," Phys. Med. Biol., vol. 43, pp. 847-856, 1998.

[16] J. Radon, "Über die Bestimmung von Funktionen durch ihre Integralwerte längs gewisser Mannigfaltigkeiten," Berichte Sächsische Akademie der Wissenschaft, Math.Phys, Kl., vol. 69, pp. 262-267, 1917.
[17] P. Grangeat, Mathematical Framework of Cone-Beam 3D-Reconstruction via the First Derivative of the Radon Transformation. Berlin, Germany: Springer, 1991.

[18] M. Davison, "A cone-beam reconstruction algorithm using shift-variant filtering and cone-beam backprojection," IEEE Trans. Med. Imag., vol. 13, pp. 186-195, Jan. 1994

[19] R. Clack and M. Defrise, "Overview of reconstruction algorithms for exact cone-beam tomography," in Proc. SPIE Conf., San Diego, CA, 1994, pp. 230-241.

[20] M. Defrise and R. Clack, "Filtered backprojection reconstruction of combined parallel beam and cone beam SPECT data," Phys. Med. Biol., vol. 40, pp. 1517-1537, 1995.

[21] P. Grangeat, Indirect Cone-Beam Three-Dimensional Image Reconstruction. New York: IOS Press, 1997.

[22] F. Natterer, The Mathematics of Computerized Tomography. New York: Wiley, 1986.

[23] P. Grangeat, R. Guillemaund, P. Rizo, R. Sauze, Q. Donner, and J. P. Gorius, "Cone-beam SPECT with a tilted detector," Comput. Med. Imag. Graph., vol. 17, no. 5, pp. 279-287, 1993.

[24] H. Kudo and T. Saito, "Derivation and implementation of a cone-beam reconstruction algorithm for nonplanar orbits," IEEE Trans. Med. Imag. vol. 13, pp. 196-211, 1994.

[25] H. K. Tuy, "An inversion formula for cone-beam reconstructions," SIAM J. Appl. Math., vol. 43, no. 3, pp. 546-552, 1983.

[26] B. D. Smith, "Image reconstruction from cone-beam projections: Necessary and sufficient conditions and reconstruction methods," IEEE Trans. Med. Imag., vol. MI-4, pp. 14-25, Jan. 1985.

[27] H. Kudo, F. Noo, and M. Defrise, "Cone-beam filtered-backprojection algorithm for truncated helical data," Phys. Med. Biol., vol. 43, pp. $2885-2909,1998$

[28] S. S. Orlov, "Theory of three-dimensional reconstruction. II. The recovery operator," Sov. Phys. Crystallogr., vol. 20, no. 4, pp. 429-433, 1975.

[29] M. Grass, T. Köhler, and R. Proksa, "3D cone-beam CT reconstruction for circular trajectories," Phys. Med. Biol., vol. 45, no. 2, pp. 329-347, 2000.

[30] M. Magnusson, "Linogram and other direct fourier methods for tomographic reconstruction,” Tech. Rep. Ph.D. thesis 320, Linköping Univ., Sweden, 1993. 